\title{
Pacific
}

Journal of

Mathematics

\section{QUANTUM GROUPS AND GENERALIZED CIRCULAR} ELEMENTS

MiCHAEL BRANNAN AND KAY KIRKPATRICK 


\title{
QUANTUM GROUPS AND GENERALIZED CIRCULAR ELEMENTS
}

\author{
MICHAEL BRANNAN AND KAY KIRKPATRICK
}

\begin{abstract}
We show that with respect to the Haar state, the joint distributions of the generators of Van Daele and Wang's free orthogonal quantum groups are modeled by free families of generalized circular elements and semicircular elements in the large (quantum) dimension limit. We also show that this class of quantum groups acts naturally as distributional symmetries of almostperiodic free Araki-Woods factors.
\end{abstract}

\section{Introduction}

There are intriguing connections between the representation theory of certain classes of compact matrix groups and independent Gaussian structures in probability theory. For instance, if one considers the $N^{2}$ matrix elements $\left\{u_{i j}\right\}_{1 \leq i, j \leq N}$ of the fundamental representation of the $N \times N$ orthogonal group $O_{N}=O_{N}(\mathbb{R})$ on the Hilbert space $\mathbb{C}^{N}$, then it is well known that the joint moments of these variables with respect to the Haar probability measure are approximated by an independent and identically distributed, mean zero, variance $1 / N$ family of real Gaussian random variables in the large $N$ limit; see, for example, [Diaconis and Freedman 1987]. Intimately related to this asymptotic Gaussianity result is the celebrated theorem of Freedman [1962; Diaconis and Freedman 1987], which says that an infinite sequence $\boldsymbol{x}=\left(x_{n}\right)_{n \in \mathbb{N}}$ of real-valued random variables is a conditionally independent centered Gaussian family with common variance if and only if the sequence is rotatable: i.e., for each $N \in \mathbb{N}$, the joint distribution of the $N$-dimensional truncation $\boldsymbol{x}_{N}=\left(x_{n}\right)_{1 \leq n \leq N}$ of $\boldsymbol{x}$ is invariant under rotations by $O_{N}$.

When one replaces the family of orthogonal groups $\left\{O_{N}\right\}_{N \in \mathbb{N}}$ by the unitary groups $\left\{U_{N}\right\}_{N \in \mathbb{N}}$, analogous results are known to hold where one replaces real Gaussian random variables by their complex-valued counterparts. The key ingredient for the above results is a certain asymptotic orthonormality property for canonical generators (weighted Brauer diagrams, in fact) of the spaces of intertwiners between the tensor powers of the fundamental representations of these groups in the large rank limit. This asymptotic feature of the representation theory is most concisely

MSC2010: primary 20G42, 46L54; secondary 46L65.

Keywords: quantum groups, free probability, free Araki-Woods factor, free quasifree state. 
expressed via the so-called Weingarten calculus developed in [Collins 2003; Collins and Śniady 2006], with origins in the pioneering work of Weingarten [1978] on the asymptotics of unitary matrix integrals. For a broad treatment of probabilistic symmetries, we refer to the text [Kallenberg 2005].

Within the framework of operator algebras and noncommutative geometry, compact quantum groups provide a vast and rich generalization of the theory of compact groups. The operator algebraic theory of compact quantum groups was pioneered by Woronowicz — see [1987; 1998], for instance — and has led recently to many interesting examples and developments in the theory of operator algebras.

One can ask noncommutative probabilistic questions about compact quantum groups, because every compact quantum group $\mathbb{G}$ admits a natural analogue of the Haar probability measure (the Haar state). The last decade or so has seen a flurry of activity in this direction, particularly for free quantum groups and Voiculescu's free probability theory. For instance, the free orthogonal quantum groups $O_{N}^{+}$ and free unitary quantum groups $U_{N}^{+}$discovered by Wang [1993] turn out to have interesting noncommutative probabilistic structures that share deep parallels with the aforementioned classical results for $O_{N}$ and $U_{N}$. Most notable for our purposes are the works of Banica-Collins [2007] and Curran [2010]. Banica and Collins show that the rescaled matrix elements $\left\{\sqrt{N} u_{i j}\right\}_{1 \leq i, j \leq N}$ of the fundamental representation of $O_{N}^{+}$(respectively $U_{N}^{+}$) converge in joint distribution to a freely independent family of standard - mean zero, variance one - semicircular (respectively circular) elements in a free group factor. Curran provides a free probability analogue of Freedman's rotatability theorem: An infinite sequence $\boldsymbol{x}=\left(x_{n}\right)_{n \in \mathbb{N}}$ of self-adjoint noncommutative random variables in a $\mathrm{W}^{*}$-probability space $(M, \varphi)$ is quantum rotatable if and only if there exists a $\mathrm{W}^{*}$-subalgebra $B \subseteq M$ and a $\varphi$-preserving conditional expectation $E: M \rightarrow B$ such that $\boldsymbol{x}$ is an identically distributed family of mean zero semicircular elements that is free with amalgamation over $B$. A similar result for $U_{N}^{+}$is obtained in [loc. cit.].

In this paper, we consider similar noncommutative probabilistic questions for a broad class of compact quantum groups introduced by Van Daele and Wang [1996] that generalizes the construction of $U_{N}^{+}$and $O_{N}^{+}$(which they called universal quantum groups). To define such a universal quantum group, one uses an invertible matrix $F \in \mathrm{GL}_{N}(\mathbb{C})$ to deform the defining algebraic relations for the quantum groups $O_{N}^{+}$and $U_{N}^{+}$, yielding a pair of new compact matrix quantum groups called $O_{F}^{+}$and $U_{F}^{+}$. When $F=1$ (the $N \times N$ identity matrix), we recover $O_{N}^{+}$and $U_{N}^{+}$ as special cases (see Section 3 for precise definitions). We follow recent literature conventions and refer to $O_{F}^{+}$as free orthogonal quantum groups and $U_{F}^{+}$as free unitary quantum groups (both with parameter matrix $F$ ).

The quantum groups $\mathbb{G}=O_{F}^{+}, U_{F}^{+}$(at least when $F$ is not a multiple of a unitary matrix) are especially interesting because their Haar states are nontracial 
and because the corresponding quantum group von Neumann algebras $L^{\infty}(\mathbb{G})$ are known to be type III factors in many cases; see [De Commer et al. 2014; Vaes and Vergnioux 2007].

The main result of this paper is that in this far more general (possibly nontracial) setting, asymptotic freeness still emerges in the large rank limit. In Theorems 5.1, 5.2, and 5.4, we show that the joint distribution of the (suitably rescaled) matrix elements of the fundamental representation of the quantum group $O_{F}^{+}$can be approximated by a freely independent family of noncommutative random variables consisting of semicircular elements and Shlyakhtenko's generalized circular elements [1997], which is built in a natural way from the initial data $F \in \mathrm{GL}_{N}(\mathbb{C})$. Generalized circular elements are nontracial deformations of Voiculescu's circular elements, and they arise as canonical generators of free Araki-Woods factors [loc. cit.]. Since free Araki-Woods factors are the natural nontracial (or type III) deformations of the free group factors, our asymptotic freeness results for $O_{F}^{+}$ provide a satisfactory generalization of the tracial asymptotic freeness results of [Banica and Collins 2007]. We also remark in Section 5C how similar asymptotic freeness results can be obtained for the free unitary quantum groups $U_{F}^{+}$.

Our proofs of asymptotic freeness results in $O_{F}^{+}$and $U_{F}^{+}$follow the general outline of the earlier work [Banica and Collins 2007; Collins and Śniady 2006], and we use a modified version of the Weingarten calculus for our situation. In our case, the formulas become a bit more unwieldy, a consequence of the extra parameters that arise from the nontrivial matrix $F \in \mathrm{GL}_{N}(\mathbb{C})$. On the other hand, there is one significant and interesting difference between our (nontracial) setting and the earlier asymptotic (free) independence results on groups and quantum groups where the Haar state is tracial. In the case of $O_{F}^{+}$and $U_{F}^{+}, F \in \mathrm{GL}_{N}(\mathbb{C})$, we find that the error in approximation of joint moments by free variables is of order

$$
O\left(\left(\operatorname{Tr}\left(F^{*} F\right)\right)^{-1}\right),
$$

a bound that is in many cases much smaller than the traditional bound given by $O(1 / N)$ in the classical case. This fact allows us to observe, for example, asymptotic freeness results in a fixed dimension $N$, by considering families of quantum groups $\left\{O_{F}^{+}\right\}_{F \in \Lambda \subset \mathrm{GL}_{N}(\mathbb{C})}$ where the quantum dimension $\operatorname{Tr}\left(F^{*} F\right)$ tends to infinity; see Theorem 5.4.

Based on our nontracial asymptotic freeness results described above, together with Curran's work on quantum rotatability [2010], it now becomes natural to ask whether the free quantum groups $O_{F}^{+}$act nontrivially on free Araki-Woods factors in a free-quasifree state-preserving way. In Section 6, we answer this question in the affirmative, and as a result we observe that almost-periodic free Araki-Woods factors admit a wealth of quantum symmetries. A future goal of the authors is to find a suitable "type III" version of Freedman's theorem adapted to $O_{F}^{+}, U_{F}^{+}$, 
and free Araki-Woods factors. After a first version of this paper appeared, it was pointed out to the authors that the main result of Section 6, namely Theorem 6.5, can also be obtained as a special case of a very general result of S. Vaes [2005, Proposition 3.1].

We finish this section with an outline of the paper's organization. Section 2 discusses some preliminaries on quantum groups and free probability that are required. Section 3 defines the quantum groups $O_{F}^{+}$and $U_{F}^{+}$and gives Weingartentype formulas for joint moments of the generators of $O_{F}^{+}$with respect to the Haar state. Section 4 considers the large quantum dimension asymptotics of these Weingarten formulas. Section 5 gives various asymptotic freeness results for $O_{F}^{+}$, and includes a remark on how to extend our results on $O_{F}^{+}$to some of their unitary counterparts $U_{F}^{+}$. Finally Section 6 considers $O_{F}^{+}$as quantum symmetries of almostperiodic free Araki-Woods factors. This is achieved by associating to each $O_{F}^{+}$a canonical free family of generalized circular elements whose joint distribution is invariant under quantum rotations by $O_{F}^{+}$.

\section{Preliminaries}

In this section we briefly review some concepts from free probability theory and compact quantum group theory. For more details, we refer the reader to [Nica and Speicher 2006] for free probability and to [Timmermann 2008; Woronowicz 1998] for quantum groups.

2A. Noncommutative probability spaces and free independence. A noncommutative probability space $(N C P S)$ is a pair $(A, \varphi)$, where $A$ is a unital $C^{*}$-algebra, and $\varphi: A \rightarrow \mathbb{C}$ is a state (i.e., a linear functional such that $\varphi\left(1_{A}\right)=1$ and $\varphi\left(a^{*} a\right) \geq 0$ for all $a \in A$ ). Elements $a \in A$ are called random variables. Given a family of random variables $X=\left\{x_{r}\right\}_{r \in \Lambda} \subset(A, \varphi)$, the joint distribution of $X$ is the collection of all joint $*$-moments

$$
\left\{\varphi\left(P\left(\left(x_{r}\right)_{r \in \Lambda}\right)\right): P \in \mathbb{C}\left\langle t_{r}, t_{r}^{*}: r \in \Lambda\right\rangle\right\},
$$

where $\mathbb{C}\left\langle t_{r}, t_{r}^{*}: r \in \Lambda\right\rangle$ is the unital $*$-algebra of noncommutative polynomials in the variables $\left\{t_{r}\right\}_{r \in \Lambda}$, equipped with antilinear involution $t_{r} \mapsto t_{r}^{*}$. Given another family of random variables $Y=\left\{y_{r}\right\}_{r \in \Lambda}$ in a NCPS $(B, \psi)$, we say that $X$ and $Y$ are identically distributed if

$$
\varphi\left(P\left(\left(x_{r}\right)_{r \in \Lambda}\right)\right)=\psi\left(P\left(\left(y_{r}\right)_{r \in \Lambda}\right)\right) \quad \text { for all } P \in \mathbb{C}\left\langle t_{r}, t_{r}^{*}: r \in \Lambda\right\rangle .
$$

Let $(A, \varphi)$ be a NCPS. A family of $*$-subalgebras $\left\{A_{r}\right\}_{r \in \Lambda}$ of $A$ is said to be freely independent (or simply free) if the following condition holds: for any choice of indices $r(1) \neq r(2), r(2) \neq r(3), \ldots, r(k-1) \neq r(k) \in \Lambda$ and any choice of 
centered random variables $x_{r(j)} \in A_{r(j)}$ (i.e., $\left.\varphi\left(x_{r(j)}\right)=0\right)$, we have the equality

$$
\varphi\left(x_{r(1)} x_{r(2)} \ldots x_{r(k)}\right)=0 .
$$

A family of random variables $X=\left\{x_{r}\right\}_{r \in \Lambda} \subset(A, \varphi)$ is said to be free if the family of unital $*$-subalgebras

$$
\left\{A_{r}\right\}_{r \in \Lambda}, \quad A_{r}:=\operatorname{alg}\left(1, x_{r}, x_{r}^{*}\right),
$$

is free in the above sense. Let $S_{\alpha}=\left\{x_{r}^{(\alpha)}\right\}_{r \in \Lambda} \subset\left(A_{\alpha}, \varphi_{\alpha}\right)$ be a net of families of random variables and $S=\left\{x_{r}\right\}_{r \in \Lambda} \in(A, \varphi)$ be another family of random variables. We say that $S_{\alpha}$ converges to $S$ in distribution (and write $S_{\alpha} \rightarrow S$ ) if, for any noncommutative polynomial $P \in \mathbb{C}\left\langle X_{r}: r \in \Lambda\right\rangle$,

$$
\lim _{\alpha} \varphi_{\alpha}\left(P\left(S_{\alpha}\right)\right)=\varphi(P(S)) .
$$

2B. Fock spaces, semicircular elements, and generalized circular elements. Let $H$ be a complex Hilbert space. The full Fock space is the Hilbert space

$$
\mathcal{F}(H)=\bigoplus_{n=0}^{\infty} H^{\otimes n},
$$

where we put $H^{\otimes 0}:=\mathbb{C} \Omega$, where $\Omega$ is a fixed unit vector, called the vacuum vector. The vacuum expectation is the state $\varphi_{\Omega}: \mathcal{B}(\mathcal{F}(H)) \rightarrow \mathbb{C}$ given by $\varphi_{\Omega}(x)=\langle\Omega \mid x \Omega\rangle$, $x \in \mathcal{B}(\mathcal{F}(H))$.

For each $\xi \in H$, we define the left creation operator $\ell(\xi) \in \mathcal{B}(\mathcal{F}(H))$ by

$$
\left\{\begin{aligned}
\ell(\xi) \Omega & =\xi \\
\ell(\xi) \eta & =\xi \otimes \eta, \quad \eta \in H^{\otimes n}, n \geq 1 .
\end{aligned}\right.
$$

Note that $\|\ell(\xi)\|_{\mathcal{B}(\mathcal{F}(H))}=\|\xi\|_{H}$. Given a NCPS $(A, \varphi)$, a (standard) semicircular element is a self-adjoint random variable $x \in A$ with the same distribution as $s(\xi):=\ell(\xi)+\ell(\xi)^{*} \in\left(\mathcal{B}(\mathcal{F}(H)), \varphi_{\Omega}\right)$, where $\xi \in H$ is a unit vector. Given $\alpha, \beta \in \mathbb{R}^{+}$, a random variable $x \in(A, \varphi)$ is called an $(\alpha, \beta)$-generalized circular element if it has the same distribution as the element $\alpha \ell(\xi)+\beta \ell(\eta)^{*} \in\left(\mathcal{B}(\mathcal{F}(H)), \varphi_{\Omega}\right)$, where $\xi, \eta$ are orthonormal vectors in $H$. One can readily verify that for an $(\alpha, \beta)$-generalized circular element $x$,

$$
\varphi\left(x^{*} x\right)=\alpha^{2} \quad \text { and } \quad \varphi\left(x x^{*}\right)=\beta^{2},
$$

and this information completely determines the $*$-moments of $x$ with respect to $\varphi$. We will call the numbers $\alpha^{2}$ and $\beta^{2}$ the left and right variances of $x$, respectively.

Next, we want to state a well known theorem which gives a combinatorial characterization of the joint distribution of a free semicircular or generalized circular 
family. To do this, we first need some notation concerning noncrossing partitions that will be used below and throughout the remainder of the paper.

Notation 2.1. Let $k \in \mathbb{N}$ and denote by $[k]$ the ordered set $\{1, \ldots, k\}$.

(1) The lattice of partitions of $[k]$ will be denoted by $\mathcal{P}(k)$, and the lattice of noncrossing partitions will be denoted by $\mathcal{N C}(k)$. The standard partial order on both lattices will be denoted by $\leq$.

(2) If $\pi \in \mathcal{P}(k)$ partitions [k] into $r$ disjoint, nonempty subsets $\mathcal{V}_{1}, \ldots, \mathcal{V}_{r}$ (called blocks), we write $|\pi|=r$ and say that $\pi$ has $r$ blocks.

(3) Given a function $i:[k] \rightarrow \Lambda$, we denote by ker $i$ the element of $\mathcal{P}(k)$ whose blocks are the equivalence classes of the relation

$$
s \sim_{\text {ker } i} t \quad \Longleftrightarrow \quad i(s)=i(t) .
$$

Note that if $\pi \in \mathcal{P}(k)$, then $\pi \leq$ ker $i$ is equivalent to the condition that whenever $s$ and $t$ are in the same block of $\pi, i(s)$ must equal $i(t)$ (i.e., the function $i:[k] \rightarrow \Lambda$ is constant on the blocks of $\pi$ ).

(4) Elements of $\mathcal{P}(k)$ which partition $[k]$ into subsets with exactly two elements are called pairings and the set of pairings of $[k]$ is denoted by $\mathcal{P}_{2}(k)$. We also write $\mathcal{N C}_{2}(k)=\mathcal{P}_{2}(k) \cap \mathcal{N C}(k)$. If $k$ is odd, we of course have $\mathcal{P}_{2}(k)=\mathcal{N C}_{2}(k)=\varnothing$.

(5) Given $\pi \in \mathcal{P}_{2}(k)$ and $s, t \in[k]$, we will always write $(s, t) \in \pi$ if $\{s, t\}$ is a block of $\pi$ and $s<t$.

(6) Let $\epsilon:[k] \rightarrow\{1, *\}$ be a function. We let $\mathcal{N C}_{2}^{\epsilon}(k) \subset \mathcal{N C}_{2}(k)$ be the subset of all noncrossing pairings such that for all $(s, t) \in \pi$,

$$
\epsilon(s) \neq \epsilon(t) .
$$

Theorem 2.2 [Nica and Speicher 2006, Chapters 7 and 15]. Let $X=\left(x_{r}\right)_{r \in \Lambda}$ be $a$ family of random variables in an NCPS $(A, \varphi)$.

(1) If $x_{r}=x_{r}^{*}$ for each $r \in \Lambda$, then $X$ is a free family of standard semicircular variables if and only if for any $k \in \mathbb{N}$ and $r:[k] \rightarrow \Lambda$,

$$
\begin{aligned}
\varphi\left(x_{r(1)} \cdots x_{r(k)}\right) & =\sum_{\substack{\pi \in \mathcal{N} \mathcal{C}_{2}(k) \\
\operatorname{ker} r \geq \pi}} \prod_{(s, t) \in \pi} \varphi\left(x_{r(s)} x_{r(t)}\right) \\
& =\left|\left\{\pi \in \mathcal{N C}_{2}(k): \operatorname{ker} r \geq \pi\right\}\right| .
\end{aligned}
$$

(2) Let $\left(\alpha_{r}, \beta_{r}\right)_{r \in \Lambda} \subset \mathbb{R}^{+} \times \mathbb{R}^{+}$. Then $X$ is a free family of $\left(\alpha_{r}, \beta_{r}\right)$-generalized circular elements if and only if for any $k \in \mathbb{N}, r:[k] \rightarrow \Lambda$, and $\epsilon:[k] \rightarrow\{1, *\}$,

$$
\varphi\left(x_{r(1)}^{\epsilon(1)} \cdots x_{r(k)}^{\epsilon(k)}\right)=\sum_{\substack{\pi \in \mathcal{N} \mathcal{C}_{2}^{\epsilon}(k) \\ \operatorname{ker} r \geq \pi}} \prod_{(s, t) \in \pi} \varphi\left(x_{r(s)}^{\epsilon(s)} x_{r(t)}^{\epsilon(t)}\right)
$$


where, in the above equation,

$$
\varphi\left(x_{r(s)}^{\epsilon(s)} x_{r(t)}^{\epsilon(t)}\right)= \begin{cases}\alpha_{r(s)}^{2}, & (\epsilon(s), \epsilon(t))=(*, 1) \\ \beta_{r(s)}^{2}, & (\epsilon(s), \epsilon(t))=(1, *)\end{cases}
$$

2C. Free Araki-Woods factors and generalized circular elements. Let $H_{\mathbb{R}}$ be a real separable Hilbert space and let $\left(U_{t}\right)$ be an orthogonal representation of $\mathbb{R}$ on $H_{\mathbb{R}}$. Let $H=H_{\mathbb{R}} \otimes_{\mathbb{R}} \mathbb{C}$ be the complexified Hilbert space. If $A$ is the infinitesimal generator of (the extension of) $U_{t}$ on $H$ (i.e., $U_{t}=A^{i t}$ ), then it follows that the map $j: H_{\mathbb{R}} \hookrightarrow H$ defined by $j(\xi)=\left(2 /\left(A^{-1}+1\right)\right)^{1 / 2} \xi$ is an isometric embedding of $H_{\mathbb{R}}$ into $H$ [Shlyakhtenko 1997]. Let $K_{\mathbb{R}}=j\left(H_{\mathbb{R}}\right)$; then $K_{\mathbb{R}} \cap i K_{\mathbb{R}}=\{0\}$ and $K_{\mathbb{R}}+i K_{\mathbb{R}}$ is dense in $H$. The free Araki-Woods factor is the von Neumann algebra

$$
\Gamma\left(H_{\mathbb{R}}, U_{t}\right)^{\prime \prime}=\mathrm{W}^{*}\left(\ell(\xi)+\ell(\xi)^{*}: \xi \in K_{\mathbb{R}}\right) \subseteq \mathcal{B}(\mathcal{F}(H)) .
$$

The restriction of the vacuum expectation $\varphi_{\Omega}=\langle\Omega \mid \cdot \Omega\rangle$ on $\mathcal{B}(\mathcal{F}(H))$ to $\Gamma\left(H_{\mathbb{R}}, U_{t}\right)^{\prime \prime}$ is always a faithful normal state, and turns $\left(\Gamma\left(H_{\mathbb{R}}, U_{t}\right)^{\prime \prime}, \varphi_{\Omega}\right)$ into a noncommutative probability space.

We recall from [loc. cit.] that $U_{t}$ is the trivial representation if and only if $\Gamma\left(H_{\mathbb{R}}, U_{t}\right)^{\prime \prime} \cong L\left(\mathbb{F}_{\operatorname{dim} H_{\mathbb{R}}}\right)$, the von Neumann algebra generated by the left regular representation of the free group on $\operatorname{dim} H_{\mathbb{R}}$ generators. Otherwise, $\Gamma\left(H_{\mathbb{R}}, U_{t}\right)^{\prime \prime}$ is a type III factor.

Free Araki-Woods factors arise naturally when one considers free families of generalized circular elements that we introduced earlier. More precisely, we have the following theorem, which follows easily from the results in [op. cit., Section 6].

Theorem 2.3 [Shlyakhtenko 1997]. Let $X=\left(x_{r}\right)_{r \in \Lambda}$ be a free family of $\left(\alpha_{r}, \beta_{r}\right)$ generalized circular elements in a noncommutative probability space $(A, \varphi)$ and let $0<\lambda_{r}=\min \left\{\alpha \beta^{-1}, \beta \alpha^{-1}\right\} \leq 1$. Then there is a state-preserving $*$-isomorphism $\left(\mathrm{W}^{*}(X), \varphi\right) \cong\left(\Gamma\left(H_{\mathbb{R}}, U_{t}\right)^{\prime \prime}, \varphi_{\Omega}\right)$, where $U_{t}$ is the almost-periodic orthogonal representation acting on the Hilbert space $H_{\mathbb{R}}=\bigoplus_{r \in \Lambda} \mathbb{R}^{2}$ given by

$$
U_{t}=\bigoplus_{r \in \Lambda} R_{\lambda_{r}}(t), \quad \text { where } R_{\lambda_{r}}(t)=\left(\begin{array}{rr}
\cos \left(t \log \lambda_{r}\right) & -\sin \left(t \log \lambda_{r}\right) \\
\sin \left(t \log \lambda_{r}\right) & \cos \left(t \log \lambda_{r}\right)
\end{array}\right) .
$$

Moreover, every free Araki-Woods factor $\Gamma\left(H_{\mathbb{R}}, U_{t}\right)^{\prime \prime}$ arising from an almostperiodic representation $U_{t}$ arises in this fashion.

2D. Compact quantum groups. A compact quantum group $\mathbb{G}$ is a pair $(C(\mathbb{G}), \Delta)$ where $C(\mathbb{G})$ is a unital $C^{*}$-algebra and $\Delta: C(\mathbb{G}) \rightarrow C(\mathbb{G}) \otimes C(\mathbb{G})$ is a unital 
*-homomorphism satisfying

$$
\left\{\begin{aligned}
(\iota \otimes \Delta) \Delta & =(\Delta \otimes \iota) \Delta & & \text { (coassociativity), } \\
{[\Delta(C(\mathbb{G}))(1 \otimes C(\mathbb{G}))] } & =[\Delta(C(\mathbb{G}))(C(\mathbb{G}) \otimes 1)] & & \\
& =C(\mathbb{G}) \otimes C(\mathbb{G}) & & \text { (nondegeneracy), }
\end{aligned}\right.
$$

where $[S]$ denotes the norm-closed linear span of a subset $S \subset C(\mathbb{G}) \otimes C(\mathbb{G})$. Here and in the rest of the paper, the symbol $\otimes$ will denote the minimal tensor product of $\mathrm{C}^{*}$-algebras, $\bar{\otimes}$ will denote the spatial tensor product of von Neumann algebras, and $\odot$ will denote the algebraic tensor product of complex associative algebras. The homomorphism $\Delta$ is called a coproduct.

For any compact quantum group $\mathbb{G}=(C(\mathbb{G}), \Delta)$, there exists a unique Haar state $h_{\mathbb{G}}: C(\mathbb{G}) \rightarrow \mathbb{C}$ which satisfies the following left and right $\Delta$-invariance property, for all $a \in C(\mathbb{G})$ :

$$
\left(h_{\mathbb{G}} \otimes \iota\right) \Delta(a)=\left(\iota \otimes h_{\mathbb{G}}\right) \Delta(a)=h_{\mathbb{G}}(a) 1 .
$$

Note that in general $h=h_{\mathbb{G}}$ is not faithful on $C(\mathbb{G})$. In any case, we can construct a GNS representation $\pi_{h}: C(\mathbb{G}) \rightarrow \mathcal{B}\left(L^{2}(\mathbb{G})\right)$, where $L^{2}(\mathbb{G})$ is the Hilbert space obtained by separation and completion of $C(\mathbb{G})$ with respect to the sesquilinear form $\langle a \mid b\rangle=h\left(a^{*} b\right)$, and $\pi_{h}$ is the natural extension to $L^{2}(\mathbb{G})$ of the left multiplication action of $C(\mathbb{G})$ on itself. The von Neumann algebra of $\mathbb{G}$ is given by

$$
L^{\infty}(\mathbb{G})=\pi_{h}(C(\mathbb{G}))^{\prime \prime} \subseteq \mathcal{B}\left(L^{2}(\mathbb{G})\right) .
$$

We note that $\Delta_{r}$ extends to an injective normal $*$-homomorphism $\Delta_{r}: L^{\infty}(\mathbb{G}) \rightarrow$ $L^{\infty}(\mathbb{G}) \bar{\otimes} L^{\infty}(\mathbb{G})$, and the Haar state on $C(\mathbb{G})$ lifts to a faithful normal $\Delta_{r}$-invariant state on $L^{\infty}(\mathbb{G})$.

Let $H$ be a finite dimensional Hilbert space and $U \in \mathcal{B}(H) \otimes C(\mathbb{G})$ be invertible (unitary). Then $U$ is called a (unitary) representation of $\mathbb{G}$ if, following the leg numbering convention,

$$
(\iota \otimes \Delta) U=U_{12} U_{13}
$$

If we fix an orthonormal basis of $H$, we can identify $U$ with an invertible matrix $U=\left[u_{i j}\right] \in M_{N}(C(\mathbb{G}))$ and (2-2) means exactly that

$$
\Delta\left(u_{i j}\right)=\sum_{k=1}^{N} u_{i k} \otimes u_{k j} \quad(1 \leq i, j \leq N) .
$$

Of course the unit $1 \in C(\mathbb{G})$ is always a representation of $\mathbb{G}$, called the trivial representation.

Let $U \in \mathcal{B}\left(H_{1}\right) \otimes C(\mathbb{G})$ and $V \in \mathcal{B}\left(H_{2}\right) \otimes C(\mathbb{G})$ be two representations of $\mathbb{G}$. An intertwiner between $U$ and $V$ is a bounded linear map $T: H_{1} \rightarrow H_{2}$ such that 
$(T \otimes \imath) U=V(T \otimes \imath)$. The Banach space of all such intertwiners is denoted by $\operatorname{Hom}_{\mathbb{G}}(U, V)$. When $U=1$ is the trivial representation, we write $\operatorname{Hom}_{\mathbb{G}}(U, V)=$ $\operatorname{Fix}(V) \subset H_{2}$, and call $\operatorname{Fix}(V)$ the space of fixed vectors for $V$. If there exists an invertible (unitary) intertwiner between $U$ and $V$, they are said to be (unitarily) equivalent. A representation is said to be irreducible if its only self-intertwiners are the scalar multiples of the identity map. It is known that each irreducible representation of $\mathbb{G}$ is finite dimensional and every finite dimensional representation is equivalent to a unitary representation. In addition, every unitary representation is unitarily equivalent to a direct sum of irreducible representations.

A compact quantum group $\mathbb{G}$ is called a compact matrix quantum group if there exists a finite dimensional unitary representation $U=\left[u_{i j}\right] \in M_{N}(C(\mathbb{G}))$ whose matrix elements generate $C(\mathbb{G})$ as a $C^{*}$-algebra. Such a representation $U$ is called a fundamental representation of $\mathbb{G}$. In this case, we note that the Haar state $h$ is faithful when restricted to the dense unital $*$-subalgebra $\operatorname{Pol}(\mathbb{G}) \subseteq C(\mathbb{G})$ generated by $\left\{u_{i j}\right\}_{1 \leq i, j \leq N}$.

\section{The free quantum groups $O_{F}^{+}$and $U_{F}^{+}$}

In this section we recall the definition of the free orthogonal and unitary quantum groups $O_{F}^{+}$and $U_{F}^{+}$, introduced by Van Daele and Wang in [1996].

Notation 3.1. Given a complex $*$-algebra $A$ and a matrix $X=\left[x_{i j}\right] \in M_{N}(A)$, we denote by $\bar{X}$ the matrix $\left[x_{i j}^{*}\right] \in M_{N}(A)$.

Definition 3.2 [Van Daele and Wang 1996]. Let $N \geq 2$ be an integer and let $F \in \mathrm{GL}_{N}(\mathbb{C})$.

(1) The free unitary quantum group $U_{F}^{+}$(with parameter matrix $F$ ) is the compact quantum group given by the universal $C^{*}$-algebra

$$
C\left(U_{F}^{+}\right)=\mathrm{C}^{*}\left(\left\{v_{i j}\right\}_{1 \leq i, j \leq N}: V=\left[v_{i j}\right] \text { is unitary and } F \bar{V} F^{-1}\right. \text { is unitary), }
$$

together with coproduct $\Delta: C\left(U_{F}^{+}\right) \rightarrow C\left(U_{F}^{+}\right) \otimes C\left(U_{F}^{+}\right)$given by

$$
\Delta\left(v_{i j}\right)=\sum_{k=1}^{N} v_{i k} \otimes v_{k j} \quad(1 \leq i, j \leq N) .
$$

(2) Let $c= \pm 1$ and assume that $F \bar{F}=c 1$. The free orthogonal quantum group $O_{F}^{+}$ (with parameter matrix $F$ ) is the compact quantum group given by the universal $\mathrm{C}^{*}$-algebra

$$
C\left(O_{F}^{+}\right)=\mathrm{C}^{*}\left(\left\{u_{i j}\right\}_{1 \leq i, j \leq N}: U=\left[u_{i j}\right] \text { is unitary and } U=F \bar{U} F^{-1}\right),
$$


together with coproduct $\Delta: C\left(O_{F}^{+}\right) \rightarrow C\left(O_{F}^{+}\right) \otimes C\left(O_{F}^{+}\right)$given by

$$
\Delta\left(u_{i j}\right)=\sum_{k=1}^{N} u_{i k} \otimes u_{k j} \quad(1 \leq i, j \leq N) .
$$

Remark 3.3. The coproduct $\Delta$ is defined so that the matrices of generators $V=\left[v_{i j}\right]$ and $U=\left[u_{i j}\right]$ are always fundamental representations of the compact matrix quantum group $U_{F}^{+}$and $O_{F}^{+}$.

Remark 3.4. Note that the above definition for $O_{F}^{+}$makes sense for any $F \in$ $\mathrm{GL}_{N}(\mathbb{C})$. The additional condition $F \bar{F}= \pm 1$ is equivalent to the requirement that $U$ is always an irreducible representation of $O_{F}^{+}$. Indeed, Banica [1996] showed that $U$ is irreducible if and only if $F \bar{F}= \pm \lambda 1(\lambda>0)$; moreover we clearly have $O_{F}^{+}=O_{\lambda^{-1 / 2} F}^{+}$.

We remark that for our asymptotic freeness results, our assumption that $F \bar{F}= \pm I$ is not a major restriction. Indeed, by a result of Wang [2002, Section 6], $O_{F}^{+}$for generic $F \in \mathrm{GL}_{N}(\mathbb{C})$ can be decomposed into a free product of finitely many quantum groups $O_{F_{i}}^{+}$and $U_{P_{k}}^{+}$with $F_{i}, P_{k}$ invertible matrices and $F_{i} \bar{F}_{i}= \pm 1$.

For the remainder of the paper, we will deal mostly with the free orthogonal quantum groups $O_{F}^{+}$. Later on in Section $5 \mathrm{C}$ we indicate how to extend some of our orthogonal results to the unitary case.

3A. Canonical F-matrices for $\boldsymbol{O}_{F}^{+}$. Let $c \in\{ \pm 1\}$ and let $F \in \mathrm{GL}_{N}(\mathbb{C})$ be such that $F \bar{F}=c 1$. In [Bichon et al. 2006], it is shown that if $c=1$, then there is an integer $0 \leq k \leq N / 2$, a nondecreasing sequence $\rho=\left(\rho_{i}\right)_{i=1}^{k} \in(0,1)^{k}$, and a unitary $w \in U_{N}$ such that

$$
F_{\rho}^{(+1)}:=w^{t} F w=\left(\begin{array}{ccc}
0 & D_{k}(\rho) & 0 \\
D_{k}(\rho)^{-1} & 0 & 0 \\
0 & 0 & 1_{N-2 k}
\end{array}\right),
$$

where $D_{k}(\rho)$ denotes the $k \times k$ diagonal matrix with diagonal entries given by the $k$-tuple $\rho$.

On the other hand if $c=-1$, then by [loc. cit.] $N=2 k$ must be even and there must exist a nondecreasing sequence $\rho=\left(\rho_{i}\right)_{i=1}^{k} \in(0,1]^{k}$ and a unitary $w \in U_{N}$ such that

$$
F_{\rho}^{(-1)}:=w^{t} F w=\left(\begin{array}{cc}
0 & D_{k}(\rho) \\
-D_{k}(\rho)^{-1} & 0
\end{array}\right) .
$$

Remark 3.5. Note that the Kac type quantum groups $O_{N}^{+}$correspond to the case $F=1_{N}$, which is exactly the canonical deformation matrix $F_{\rho}^{(+1)}$ with $k=0$.

According to [loc. cit.], given two matrices $F_{i} \in \mathrm{GL}_{N_{i}}(\mathbb{C})$ such that $F_{i} \bar{F}_{i}=c_{i} 1$, the two free orthogonal quantum groups $O_{F_{1}}^{+}$and $O_{F_{2}}^{+}$are isomorphic if and only 
if $N_{1}=N_{2}, c_{1}=c_{2}$, and $F_{2}=v F_{1} v^{t}$ for some unitary matrix $v \in U_{N_{1}}$. The corresponding equivalence relation on such matrices has fundamental domain given by all matrices of the form $F_{\rho}^{( \pm 1)}$. As a consequence, we call such matrices $F_{\rho}^{( \pm 1)}$ canonical $F$-matrices. The canonical $F$-matrices yield the most natural coordinate system in which to represent the isomorphism equivalence class of any given $O_{F}^{+}$.

3B. Integration over $\boldsymbol{O}_{\boldsymbol{F}}^{+}$. In this section, we consider the problem of evaluating arbitrary monomials in the generators $\left\{u_{i j}\right\}_{1 \leq i, j \leq N}$ of $C\left(O_{F}^{+}\right)$with respect to the Haar state $h_{O_{F}^{+}}$.

Notation 3.6. Fix an orthonormal basis $\left\{e_{i}\right\}_{i=1}^{N}$ for $\mathbb{C}^{N}$ and $F \in \mathrm{GL}_{N}(\mathbb{C})$. Define

$$
\xi=\sum_{i=1}^{N} e_{i} \otimes e_{i} \quad \text { and } \quad \xi^{F}=(\mathrm{id} \otimes F) \xi=\sum_{i=1}^{N} e_{i} \otimes F e_{i} .
$$

For each $l \in \mathbb{N}, \pi \in \mathcal{N C}_{2}(2 l)$, and $i:[2 l] \rightarrow[N]$ define,

$$
\delta_{\pi}^{F}(i)=\prod_{(s, t) \in \pi} F_{i(t) i(s)},
$$

and put

$$
\xi_{\pi}^{F}=\sum_{i:[2 l] \rightarrow[N]} \delta_{\pi}^{F}(i) e_{i(1)} \otimes e_{i(2)} \otimes \cdots \otimes e_{i(2 l)} \in\left(\mathbb{C}^{N}\right)^{\otimes 2 l}
$$

For the purposes of integrating monomials over $O_{F}^{+}$with respect to the Haar state, we are interested in the $l$-th tensor power of the fundamental representation $U=\left[u_{i j}\right]$ of $O_{F}^{+}$,

$$
U^{\ominus l}:=\left[u_{i(1) j(1)} \cdots u_{i(l) j(l)}\right] \in \mathcal{B}\left(\left(\mathbb{C}^{N}\right)^{\otimes l}\right) \otimes C\left(O_{F}^{+}\right) .
$$

$U^{\oplus l}$ is evidently a representation of the quantum group $O_{F}^{+}$, and the following theorem of Banica describes the space of fixed vectors of these higher tensor powers of $U$.

Theorem 3.7 [Banica 1996]. Let $N \geq 2, c \in\{ \pm 1\}$, and $F \in \mathrm{GL}_{N}(\mathbb{C})$ be such that $F \bar{F}=c 1$. Then for each $l \in \mathbb{N}$,

$$
\operatorname{Fix}\left(U^{\ominus 2 l+1}\right)=\{0\},
$$

and

$$
\operatorname{Fix}\left(U^{\ominus 2 l}\right)=\operatorname{span}\left\{\xi_{\pi}^{F}: \pi \in \mathcal{N C}_{2}(2 l)\right\} .
$$

Moreover, $\left\{\xi_{\pi}^{F}\right\}_{\pi}$ is a linear basis for $\operatorname{Fix}\left(U^{\ominus 2 l}\right)$.

With the preceding theorem in hand, we now use the Weingarten calculus to describe the Haar state on $O_{F}^{+}$in terms of the Gram matrices associated to the 
bases $\left\{\xi_{\pi}^{F}\right\}_{\pi \in \mathcal{N C}_{2}(2 l)}$ of Fix $\left(U^{\ominus 2 l}\right)$. For each $l \in \mathbb{N}$, define an $\left|\mathcal{N C}_{2}(2 l)\right| \times\left|\mathcal{N C}_{2}(2 l)\right|$ matrix $G_{2 l, F}=\left[G_{2 l, F}(\pi, \sigma)\right]_{\pi, \sigma \in \mathcal{N} C_{2}(2 l)}$ by

$$
G_{2 l, F}(\pi, \sigma)=\left\langle\xi_{\pi}^{F} \mid \xi_{\sigma}^{F}\right\rangle \quad\left(\pi, \sigma \in \mathcal{N C}_{2}(2 l)\right) .
$$

Theorem 3.8. Let $N \geq 2, c \in\{ \pm 1\}$, and $F \in \mathrm{GL}_{N}(\mathbb{C})$ be such that $F \bar{F}=c 1$. Set $N_{F}:=\operatorname{Tr}\left(F^{*} F\right)$. Then for any $l \geq 1, G_{2 l, F}$ is an invertible matrix and

$$
G_{2 l, F}(\pi, \sigma)=c^{l+|\pi \vee \sigma|} N_{F}^{|\pi \vee \sigma|} \quad\left(\pi, \sigma \in \mathcal{N C}_{2}(2 l)\right),
$$

where $\pi \vee \sigma$ denotes the join of $\pi$ and $\sigma$ in the lattice $\mathcal{P}(2 l)$.

Proof. The first assertion follows from Theorem 3.7. For the second assertion, fix $\pi, \sigma \in \mathcal{N C}_{2}(2 l)$ and let $\pi \vee \sigma=\left\{\mathcal{V}_{1}, \mathcal{V}_{2}, \ldots, \mathcal{V}_{m}\right\}$, where each $\mathcal{V}_{a}$ is a block of $\pi \vee \sigma$. Then we have

$$
G_{2 l, F}(\pi, \sigma)=\sum_{i:[2 l] \rightarrow[N]} \overline{\delta_{\pi}^{F}(i)} \delta_{\sigma}^{F}(i)=\prod_{a=1}^{m}\left(\sum_{i: \mathcal{V}_{a} \rightarrow[N]} \overline{\delta_{\pi \mid}^{F}}(i) \delta_{\left.\sigma\right|_{\mathcal{V}_{a}}}^{F}(i)\right) .
$$

From the above equation we see that $G_{2 l, F}(\pi, \sigma)$ is a multiplicative function of the blocks of $\pi \vee \sigma$, and therefore it suffices to prove the theorem when $\pi \vee \sigma=1_{2 l}$.

To prove this special case of the theorem, we proceed by induction on $l$ : If $2 l=2$, then $G_{2 l, F}=\left\langle\xi^{F} \mid \xi^{F}\right\rangle=\operatorname{Tr}\left(F^{*} F\right)=N_{F}=c^{2} N_{F}$. Now assume $l \geq 2$ and that the desired result is true for all $\pi^{\prime}, \sigma^{\prime} \in \mathcal{N C}_{2}(2 l-2)$ with $\pi^{\prime} \vee \sigma^{\prime}=1_{2 l-2}$. Fix $\pi, \sigma \in \mathcal{N C}_{2}(2 l)$ such that $\pi \vee \sigma=1_{2 l}$. Since $\pi$ is noncrossing, we can fix an interval $\{r, r+1\}$ in $\pi$ and let $\{a, r\},\{b, r+1\}$ be the corresponding (unordered) pairs of $\sigma$ that connect to $r$ and $r+1$. (Note that $\sigma$ does not pair $r$ and $r+1$ because $|\pi \vee \sigma|=1$ and $l \geq 2$.) Now let $\pi^{\prime} \in \mathcal{N C}_{2}(2 l-2)$ be the pairing obtained by deleting the block $\{r, r+1\}$ from $\pi$ and let $\sigma^{\prime} \in \mathcal{N C}_{2}(2 l-2)$ be the pairing obtained by deleting the points $r, r+1$ from $\sigma$ and pairing $a$ and $b$. Note that by construction, $\pi^{\prime} \vee \sigma^{\prime}=1_{2 l-2}$.

Using the readily verified identities

$c \xi^{F}=\left(\iota \otimes\left(\xi^{F}\right)^{*} \otimes \iota\right)\left(\xi^{F} \otimes \xi^{F}\right)=\left(\left(\xi^{F}\right)^{*} \otimes \iota\right)\left(\iota \otimes \xi^{F} \otimes \iota\right) \xi^{F}=\left(\iota \otimes\left(\xi^{F}\right)^{*}\right)\left(\iota \otimes \xi^{F} \otimes \iota\right) \xi^{F}$, it easily follows that $G_{2 l, F}(\pi, \sigma)=\left\langle\xi_{\pi}^{F} \mid \xi_{\sigma}^{F}\right\rangle=c\left\langle\xi_{\pi^{\prime}}^{F} \mid \xi_{\sigma^{\prime}}^{F}\right\rangle$. We then have from our induction assumption that

$$
G_{2 l, F}(\pi, \sigma)=c\left(c^{l-1+\left|\pi^{\prime} \vee \sigma^{\prime}\right|} N_{F}\right)=c^{l+1} N_{F} .
$$

For each $l \in \mathbb{N}$, denote by $W_{2 l, F}$ the matrix inverse of $G_{2 l, F}$. In the following theorem we give a Weingarten-type moment formula for the Haar state on $O_{F}^{+}$. Compare with [Banica and Collins 2007; Banica et al. 2009], where a version of the following result is obtained in the case where $F \in \mathrm{GL}_{N}(\mathbb{R})$ and $c=1$. 
Theorem 3.9. For each pair of multi-indices $i, j:[l] \rightarrow[N]$,

$h_{O_{F}^{+}}\left(u_{i(1) j(1)} u_{i(2) j(2)} \cdots u_{i(l) j(l)}\right)=\left\{\begin{array}{cl}0 & \text { if l is odd, } \\ \sum_{\pi, \sigma \in \mathcal{N} \mathcal{C}_{2}(l)} W_{l, F}(\pi, \sigma) \overline{\delta_{\pi}^{F}(j)} \delta_{\sigma}^{F}(i) & \text { otherwise. }\end{array}\right.$

Proof. We use the fact that if $V \in \mathcal{B}(H) \otimes C(\mathbb{G})$ is a unitary representation of a compact quantum group $\mathbb{G}$ with Haar state $h$, then $P_{V}=(\mathrm{id} \otimes h)(V)$ is the orthogonal projection onto $\operatorname{Fix}(V)$. Using this fact, the quantity we are interested in is the $(i, j)$-th matrix element of the projection $P_{U \oplus l}$. Since $P_{U \oplus l}=0$ when $l$ is odd (by Theorem 3.7), the first equality is immediate.

For the second equality, assume $l \geq 2$ is even. Let $\left\{\xi_{\pi}^{F}\right\}_{\pi \in \mathcal{N C}_{2}(l)}$ be the basis for $\operatorname{Fix}\left(U^{\oplus l}\right)$ from Theorem 3.7 and define a new set $\left\{\tilde{\xi}_{\pi}^{F}\right\}_{\pi \in \mathcal{N C} C_{2}(l)} \subset \operatorname{Fix}\left(U^{\ominus l}\right)$ by

$$
\tilde{\xi}_{\pi}^{F}=\sum_{\sigma \in \mathcal{N C}_{2}(l)} \mathrm{W}_{l, F}^{1 / 2}(\pi, \sigma) \xi_{\sigma}^{F}
$$

where $\mathrm{W}_{l, F}^{1 / 2}$ is the matrix square root of $W_{l, F}$. Then $\left\{\tilde{\xi}_{\pi}^{F}\right\}_{\pi \in \mathcal{N C} C_{2}(l)}$ is an orthonormal basis for $\operatorname{Fix}\left(U^{\ominus l}\right)$ by Theorem 3.8 and

$$
P_{U}=\sum_{\pi \in \mathcal{N C}_{2}(l)}\left|\tilde{\xi}_{\pi}^{F}\right\rangle\left\langle\tilde{\xi}_{\pi}^{F}\right|
$$

Therefore,

$$
\begin{aligned}
h_{O_{F}^{+}}\left(u_{i(1) j(1)} u_{i(2) j(2)} \cdots u_{i(l) j(l)}\right. & \\
= & \left\langle e_{i} \mid P_{U \odot l} e_{j}\right\rangle \\
= & \sum_{\rho \in \mathcal{N} C_{2}(l)}\left\langle\tilde{\xi}_{\rho}^{F} \mid e_{j}\right\rangle\left\langle e_{i} \mid \tilde{\xi}_{\rho}^{F}\right\rangle \\
= & \sum_{\pi, \sigma, \rho \in \mathcal{N} C_{2}(l)} \mathrm{W}_{l, F}^{1 / 2}(\rho, \pi)\left\langle\xi_{\pi}^{F} \mid e_{j}\right\rangle \mathrm{W}_{l, F}^{1 / 2}(\rho, \sigma)\left\langle e_{i} \mid \xi_{\sigma}^{F}\right\rangle \\
= & \sum_{\pi, \sigma \in \mathcal{N} \mathcal{C}_{2}(l)} W_{l, F}(\pi, \sigma)\left\langle\xi_{\pi}^{F} \mid e_{j}\right\rangle\left\langle e_{i} \mid \xi_{\sigma}^{F}\right\rangle \\
= & \sum_{\pi, \sigma \in \mathcal{N} \mathcal{C}_{2}(l)} W_{l, F}(\pi, \sigma) \overline{\delta_{\pi}^{F}(j)} \delta_{\sigma}^{F}(i) .
\end{aligned}
$$

3C. Integrating $*$-monomials over $\boldsymbol{O}_{\boldsymbol{F}}^{+}$. Note that the defining relations for the generators $\left\{u_{i j}\right\}_{1 \leq i, j \leq N}$ of $\left(C\left(O_{F}^{+}\right), h_{O_{F}^{+}}\right)$imply that the family $\left\{u_{i j}\right\}_{1 \leq i, j \leq N}$ is selfadjoint. Moreover, taking the deformation matrix $F$ to be in canonical form, as 
defined in Section 3A, we can write

$$
F=F_{\rho}^{(c)}=\left(\begin{array}{ccc}
0 & D_{k}(\rho) & 0 \\
c D_{k}(\rho)^{-1} & 0 & 0 \\
0 & 0 & 1_{N-2 k}
\end{array}\right) .
$$

where $D_{k}(\rho)$ denotes the $k \times k$ diagonal matrix with diagonal entries given by a $k$-tuple $\rho=\left(\rho_{i}\right)_{i=1}^{k} \subset(0,1]^{k}$, and $N=2 k$ if $c=-1$. Then $F^{-1}=c F$, and we easily compute from (3-2) that

$$
\begin{aligned}
u_{i j}^{*}=(c F U F)_{i j}=c \sum_{1 \leq r, s \leq N} & F_{i r} F_{s j} u_{r s} \\
= & \left\{\begin{array}{cl}
c F_{i, i+k} F_{j+k, j} u_{i+k, j+k} & 1 \leq i, j \leq 2 k, \\
F_{i, i+k} u_{i+k, j} & 1 \leq i \leq 2 k, j>2 k, \\
F_{j+k, j} u_{i, j+k} & 1 \leq j \leq 2 k, i>2 k, \\
u_{i j} & i, j>2 k
\end{array}\right.
\end{aligned}
$$

where in the above equations we perform the additions $i \mapsto i+k, j \mapsto j+k \bmod 2 k$. Using these equations, it is easy to see that the fundamental representation $U=$ $\left[u_{i j}\right]_{1 \leq i, j \leq N}$ of $O_{F}^{+}$admits the following canonical block-matrix decomposition.

$$
U=\left(\begin{array}{ccc}
{\left[u_{a, b}\right]_{1 \leq a, b \leq k}} & {\left[u_{a, b+k}\right]_{1 \leq a, b \leq k}} & {\left[u_{a, t}\right]_{\substack{1 \leq a \leq k \\
t \geq 2 k+1}}} \\
{\left[c \rho_{a}^{-1} \rho_{b}^{-1} u_{a, b+k}^{*}\right]_{1 \leq a, b \leq k}} & {\left[\rho_{a}^{-1} \rho_{b} u_{a, b}^{*}\right]_{1 \leq a, b \leq k}} & {\left[\rho_{a}^{-1} u_{a, t}^{*}\right]_{\substack{1 \leq a \leq k \\
t \geq 2 k+1}}} \\
{\left[u_{s, b}\right]_{\substack{1 \leq b \leq k \\
s \geq 2 k+1}}} & {\left[\rho_{b} u_{s, b}^{*}\right]_{\substack{1 \leq b \leq k \\
s \geq 2 k+1}}} & {\left[u_{s, t}\right]_{s, t \geq 2 k+1}}
\end{array}\right) .
$$

Remark 3.10. From Equation (3-6), we see that the $\mathrm{C}^{*}$-algebra $C\left(O_{F}^{+}\right)$is generated by the subset

$$
\left(\left\{u_{i j}\right\}_{\substack{1 \leq i \leq k \\ 1 \leq j \leq N}} \cup\left\{u_{i j}\right\}_{\substack{1 \leq j \leq k \\ 2 k+1 \leq i \leq N}} \cup\left\{u_{i j}\right\}_{2 k+1 \leq i, j \leq N}\right) \subset\left\{u_{i j}\right\}_{1 \leq i, j \leq N} .
$$

3C1. General $*$-moments over $O_{F}^{+}$. Let $\epsilon \in\{1, *\}$ and $i, j \in[N]$. Using (3-6) (or equivalently (3-5)), we can find unique numbers $i_{\epsilon}, j_{\epsilon} \in[N]$ and $t_{F}(i, j, \epsilon) \in \mathbb{R}$ such that

$$
u_{i j}^{\epsilon}=t_{F}(i, j, \epsilon) u_{i_{\epsilon} j_{\epsilon}} .
$$

In particular, arbitrary $*$-moments in the generators $\left\{u_{i j}\right\}_{1 \leq i, j \leq N}$ can be computed using the formula of Theorem 3.9.

Proposition 3.11. Let $l \in \mathbb{N}, i, j:[l] \rightarrow[N]$, and $\epsilon:[l] \rightarrow\{1, *\}$ be given. If $l$ is odd, then

$$
h_{O_{F}^{+}}\left(u_{i(1) j(1)}^{\epsilon(1)} u_{i(2) j(2)}^{\epsilon(2)} \cdots u_{i(l) j(l)}^{\epsilon(l)}\right)=0 .
$$


If $l$ is even, then

$$
\begin{aligned}
h_{O_{F}^{+}}\left(u_{i(1) j(1)}^{\epsilon(1)} u_{i(2) j(2)}^{\epsilon(2)} \cdots\right. & \left.u_{i(l) j(l)}^{\epsilon(l)}\right) \\
& =\prod_{r=1}^{l} t_{F}(i(r), j(r), \epsilon(r)) h_{O_{F}^{+}}\left(\prod_{r=1}^{l} u_{i(r)_{\epsilon}(r)}, j(r)_{\epsilon(r)}\right) \\
& \left.=\prod_{r=1}^{l} t_{F}(i(r), j(r), \epsilon(r)) \sum_{\pi, \sigma \in \mathcal{N} C_{2}(l)} W_{l, F}(\pi, \sigma) \overline{\delta_{\pi}^{F}\left(j_{\epsilon}\right.}\right) \delta_{\sigma}^{F}\left(i_{\epsilon}\right),
\end{aligned}
$$

where $i_{\epsilon}=\left(i_{\epsilon(1)}(1), i_{\epsilon(2)}(2), \ldots, i_{\epsilon(l)}(l)\right)$ and $j_{\epsilon}=\left(j_{\epsilon(1)}(1), j_{\epsilon(2)}(2), \ldots, j_{\epsilon(l)}(l)\right)$.

The proof of this result is immediate.

3C2. Variances of the generators of $C\left(O_{F}^{+}\right)$. The simplest (nonzero) $*$-moments are the left and right covariances of the generators $\left\{u_{i j}\right\}_{1 \leq i, j \leq N} \subset C\left(O_{F}^{+}\right)$, i.e., the quantities

$$
\left\langle u_{i j} \mid u_{k l}\right\rangle_{L}=h_{O_{F}^{+}}\left(u_{i j}^{*} u_{k l}\right) \quad \text { and } \quad\left\langle u_{k l} \mid u_{i j}\right\rangle_{R}=h_{O_{F}^{+}}\left(u_{i j} u_{k l}^{*}\right) .
$$

The left and right covariances can be computed using Proposition 3.11. Alternatively, we can compute these quantities using the Schur orthogonality relations

$$
h_{O_{F}^{+}}\left(u_{i j}^{*} u_{k l}\right)=\frac{\delta_{j l}\left(Q^{-1}\right)_{k i}}{N_{F}}, \quad h_{O_{F}^{+}}\left(u_{i j} u_{k l}^{*}\right)=\frac{\delta_{i k} Q_{l j}}{N_{F}} \quad(1 \leq i, j \leq N),
$$

where

$$
Q=F^{t} \bar{F}, \quad Q^{-1}=F F^{*}, \quad \text { and } \operatorname{Tr}(Q)=\operatorname{Tr}\left(Q^{-1}\right)=N_{F} .
$$

See, for example, [Woronowicz 1998] and the paragraphs following Theorem 7.2 of [Vaes and Vergnioux 2007]. In particular, when $F=F_{\rho}^{(c)}$ is a canonical $F$-matrix as above, then the structure of $Q$ is simple:

$$
Q=\left(\begin{array}{ccc}
D_{k}(\rho)^{-2} & 0 & 0 \\
0 & D_{k}(\rho)^{2} & 0 \\
0 & 0 & 1_{N-2 k}
\end{array}\right)
$$

In particular, it follows from (3-8) that $\left\{u_{i j}\right\}_{1 \leq i, j \leq N}$ is an orthogonal system with respect to the inner products $\langle\cdot \mid \cdot\rangle_{L}$ and $\langle\cdot \mid \cdot\rangle_{R}$ induced by $h_{O_{F}^{+}}$, and a simple calculation shows that the $N \times N$ matrix of left and right variances

$$
\Phi=\left[\left(\left\langle u_{i j} \mid u_{i j}\right\rangle_{L},\left\langle u_{i j} \mid u_{i j}\right\rangle_{R}\right)\right]_{1 \leq i, j \leq N}
$$


has the following block-matrix decomposition (compare with the decomposition of the fundamental representation of $O_{F}^{+}$given by (3-6)).

$$
\begin{aligned}
\Phi= & \frac{1}{N_{F}}\left[\left(Q_{i i}^{-1}, Q_{j j}\right)\right]_{1 \leq i, j \leq N} \\
& =\frac{1}{N_{F}}\left(\begin{array}{ccc}
{\left[\left(\rho_{a}^{2}, \rho_{b}^{-2}\right)\right]_{1 \leq a, b \leq k}} & {\left[\left(\rho_{a}^{2}, \rho_{b}^{2}\right)\right]_{1 \leq a, b \leq k}} & {\left[\left(\rho_{a}^{2}, 1\right)\right]_{k \times(N-2 k)}} \\
{\left[\left(\rho_{a}^{-2}, \rho_{b}^{-2}\right)\right]_{1 \leq a, b \leq k}} & {\left[\left(\rho_{a}^{-2}, \rho_{b}^{2}\right)\right]_{1 \leq a, b \leq k}} & {\left[\left(\rho_{a}^{-2}, 1\right)\right]_{k \times(N-2 k)}} \\
{\left[\left(1, \rho_{b}^{-2}\right)\right]_{(N-2 k) \times k}} & {\left[\left(1, \rho_{b}^{2}\right)\right]_{(N-2 k) \times k}} & {[(1,1)]_{k \times k}}
\end{array}\right)
\end{aligned}
$$

\section{Large (quantum) dimension asymptotics}

Using our Weingarten formulas (Theorem 3.9 and Proposition 3.11), we can study the large (quantum) dimension asymptotics of $*$-moments over $O_{F}^{+}$. Let $F \in$ $\mathrm{GL}_{N}(\mathbb{C})$ be such that $F \bar{F}=c 1$, and let $N_{F}=\operatorname{Tr}\left(F^{*} F\right)$. We will call the number $N_{F}$ the quantum dimension of the fundamental representation $U=\left[u_{i j}\right]$ of $O_{F}^{+}$. Note that we always have $N_{F} \geq N$. The following proposition shows that the Weingarten matrices $W_{l, F}$ associated to $O_{F}^{+}$are asymptotically diagonal as the quantum dimension $N_{F}$ tends to infinity. This result should be compared with [Banica and Collins 2007, Theorem 6.1].

Theorem 4.1. For each $l \in 2 \mathbb{N}$, as $N_{F} \rightarrow \infty$,

$$
N_{F}^{l / 2} W_{l, F}(\pi, \sigma)=\delta_{\pi, \sigma}+O\left(N_{F}^{-1}\right) \quad\left(\pi, \sigma \in \mathcal{N C}_{2}(l)\right) .
$$

Proof. According to Theorem 3.8, $W_{l, F}=G_{l, F}^{-1}$ and $G_{l, F}(\pi, \sigma)=c^{l / 2+|\pi \vee \sigma|} N_{F}^{|\pi \vee \sigma|}$. Observe that $|\pi \vee \sigma|=l / 2$ if and only if $\pi=\sigma$, and $|\pi \vee \sigma| \leq l / 2-1$ otherwise. Therefore, with respect to the operator norm, we have the asymptotic formula

$$
G_{l, F}=N_{F}^{l / 2} I+O\left(N_{F}^{l / 2-1}\right)=N_{F}^{l / 2}\left(I+O\left(N_{F}^{-1}\right)\right) .
$$

Write $N_{F}^{-l / 2} G_{l, F}=I+A_{F}$, where $\left\|A_{F}\right\| \leq C_{l} N_{F}^{-1}$ for some $C_{l} \geq 0$. Then for sufficiently large $N_{F}$, we have the absolutely convergent power series expansion

$$
N_{F}^{l / 2} W_{l, F}=\left(I+A_{F}\right)^{-1}=\sum_{r=0}^{\infty}(-1)^{r} A_{F}^{r}=I+O\left(N_{F}^{-1}\right) \quad\left(N_{F} \rightarrow \infty\right) .
$$

The result now follows.

The following proposition is a consequence of Theorem 4.1 and gives an asymptotic factorization of the normalized joint moments over $O_{F}^{+}$. 
Proposition 4.2. Fix $l \in 2 \mathbb{N}$ and $i, j:[l] \rightarrow[N]$. Then there is a constant $D_{l}>0$ (depending only on $l$ ) such that

$$
\begin{array}{r}
N_{F}^{l / 2}\left|h_{O_{F}^{+}}\left(u_{i(1) j(1)} u_{i(2) j(2)} \cdots u_{i(l) j(l)}\right)-\sum_{\pi \in \mathcal{N C} C_{2}(l)} \prod_{(s, t) \in \pi} h_{O_{F}^{+}}\left(u_{i(s) j(s)} u_{i(t) j(t)}\right)\right| \\
\leq \frac{D_{l}}{N_{F}} \max _{\pi, \sigma \in \mathcal{N C} \mathcal{C}_{2}(l)}\left|\delta_{\pi}^{F}(j) \delta_{\sigma}^{F}(i)\right| .
\end{array}
$$

Proof. Using Theorem 4.1, one can find a constant $D_{l}>0$ such that

$$
\sum_{\pi, \sigma \in \mathcal{N C}_{2}(l)}\left|N_{F}^{l / 2} W_{l, F}(\pi, \sigma)-\delta_{\pi, \sigma}\right| \leq \frac{D_{l}}{N_{F}}
$$

Since it also follows from Theorem 3.9 that

$$
\begin{aligned}
\sum_{\pi \in \mathcal{N C} \mathcal{C}_{2}(l)} \prod_{(s, t) \in \pi} h_{O_{F}^{+}}\left(u_{i(s) j(s)} u_{i(t) j(t)}\right) & =\sum_{\pi \in \mathcal{N} C_{2}(l)} \prod_{(s, t) \in \pi} N_{F}^{-1} F_{i(t) i(s)} \overline{F_{j(t) j(s)}} \\
& =N_{F}^{-l / 2} \sum_{\pi \in \mathcal{N} C_{2}(l)} \delta_{\pi}^{F}(i) \overline{\delta_{\pi}^{F}(j)},
\end{aligned}
$$

we obtain

$$
\begin{gathered}
N_{F}^{l / 2}\left|h_{O_{F}^{+}}\left(u_{i(1) j(1)} u_{i(2) j(2)} \cdots u_{i(l) j(l)}\right)-\sum_{\pi \in \mathcal{N} \mathcal{C}_{2}(l)}\right| \prod_{(s, t) \in \pi} h_{O_{F}^{+}}\left(u_{i(s) j(s)} u_{i(t) j(t)}\right) \mid \\
=\left|\sum_{\pi, \sigma \in \mathcal{N C}_{2}(l)} N_{F}^{l / 2}\left(W_{l, F}(\pi, \sigma)-\delta_{\pi, \sigma}\right) \overline{\delta_{\pi}^{F}(j)} \delta_{\sigma}^{F}(i)\right| \\
\leq \frac{D_{l}}{N_{F}} \max _{\pi, \sigma \in \mathcal{N} \mathcal{C}_{2}(l)}\left|\delta_{\pi}^{F}(j) \delta_{\sigma}^{F}(i)\right| .
\end{gathered}
$$

Using Propositions 3.11 and 4.2, we obtain a similar asymptotic factorization result for $*$-moments.

Corollary 4.3. Fix $l \in 2 \mathbb{N}, \epsilon:[l] \rightarrow\{1, *\}$, and $i, j:[l] \rightarrow[N]$. Then there is a constant $D_{l}>0$ (depending only on $l$ ) such that

$$
\begin{aligned}
N_{F}^{l / 2} \mid h_{O_{F}^{+}}\left(u_{i(1) j(1)}^{\epsilon(1)} \cdots u_{i(l) j(l)}^{\epsilon(l)}\right)- & \sum_{\pi \in \mathcal{N} \mathcal{C}_{2}(l)} \prod_{(s, t) \in \pi} h_{O_{F}^{+}}\left(u_{i(s) j(s)}^{\epsilon(s)} u_{i(t) j(t)}^{\epsilon(t)}\right) \mid \\
\leq & \frac{D_{l}}{N_{F}} \max _{\pi, \sigma \in \mathcal{N} \mathcal{C}_{2}(l)}\left|\delta_{\pi}^{F}\left(j_{\epsilon}\right) \delta_{\sigma}^{F}\left(i_{\epsilon}\right)\right| \prod_{r=1}^{l}\left|t_{F}(i(r), j(r), \epsilon(r))\right| .
\end{aligned}
$$

\section{Asymptotic freeness in $O_{F}^{+}$}

We now arrive at the main asymptotic freeness results of this paper. 
Let us fix a canonical matrix

$$
F=F_{\rho}^{(c)}=\left(\begin{array}{ccc}
0 & D_{k}(\rho) & 0 \\
c D_{k}(\rho)^{-1} & 0 & 0 \\
0 & 0 & 1_{N-2 k}
\end{array}\right) \in \mathrm{GL}_{N}(\mathbb{C}),
$$

as in Section 3C. Recall from Remark 3.10 that the subset of (rescaled) matrix elements

$$
\mathcal{S}_{F}=\left\{\sqrt{N_{F}} u_{i j}\right\}_{\substack{1 \leq i \leq k \\ 1 \leq j \leq N}} \cup\left\{\sqrt{N_{F}} u_{i j}\right\}_{\substack{1 \leq j \leq k \\ 2 k+1 \leq i \leq N}} \cup\left\{\sqrt{N_{F}} u_{i j}\right\}_{2 k+1 \leq i, j \leq N}
$$

generates the $\mathrm{C}^{*}$-algebra $C\left(O_{F}^{+}\right)$. In this section we show that the set $\mathcal{S}_{F}$ is asymptotically free in the following sense.

Theorem 5.1. Let

$$
\mathcal{S}=\left\{y_{i j}\right\}_{\substack{1 \leq i \leq k \\ 1 \leq j \leq N}} \cup\left\{y_{i j}\right\}_{\substack{1 \leq j \leq k \\ 2 k+1 \leq i \leq N}} \cup\left\{y_{i j}\right\}_{2 k+1 \leq i, j \leq N}
$$

be a family of noncommutative random variables in an NCPS $(A, \varphi)$ with the following properties.

(1) $\mathcal{S}$ is freely independent.

(2) For each $i, j$, the elements $y_{i j} \in \mathcal{S}$ and $\sqrt{N_{F}} u_{i j} \in \mathcal{S}_{F}$ have the same left and right variances, given by the matrix entries of $N_{F} \Phi$ in (3-9).

(3) If either $i \leq k$ or $j \leq k$, then each $y_{i j}$ is a generalized circular element.

(4) If $2 k+1 \leq i, j \leq N$, then $y_{i j}$ is a standard semicircular element.

Then for each $l \in 2 \mathbb{N}$, there is a constant $D_{l}>0$ such that

$$
\begin{aligned}
& \left|h_{O_{F}^{+}}\left(\sqrt{N_{F}} u_{i(1) j(1)}^{\epsilon(1)} \cdots \sqrt{N_{F}} u_{i(l) j(l)}^{\epsilon(l)}\right)-\varphi\left(y_{i(1) j(1)}^{\epsilon(1)} \cdots y_{i(l) j(l)}^{\epsilon(l)}\right)\right| \\
& \leq \frac{D_{l}}{N_{F}} \max _{\pi, \sigma \in \mathcal{N} \mathcal{C}_{2}(l)}\left|\delta_{\pi}^{F}\left(j_{\epsilon}\right) \delta_{\sigma}^{F}\left(i_{\epsilon}\right)\right| \prod_{r=1}^{l}\left|t_{F}(i(r), j(r), \epsilon(r))\right|,
\end{aligned}
$$

for each $\epsilon:[l] \rightarrow\{1, *\}$ and $i, j:[l] \rightarrow[N]$.

Proof. Since $\mathcal{S}$ is a free family consisting of generalized circular elements and standard semicircular elements satisfying conditions (2)-(4) above, Theorem 2.2 gives

$$
\begin{aligned}
\varphi\left(y_{i(1) j(1)}^{\epsilon(1)} \cdots y_{i(l) j(l)}^{\epsilon(l)}\right) & =\sum_{\pi \in \mathcal{N C} C_{2}(l)} \prod_{(s, t) \in \pi} \varphi\left(y_{i(s) j(s)}^{\epsilon(s)} y_{i(t) j(t)}^{\epsilon(t)}\right) \\
& =\sum_{\pi \in \mathcal{N C} C_{2}(l)} \prod_{(s, t) \in \pi} N_{F} h_{O_{F}^{+}}\left(u_{i(s) j(s)}^{\epsilon(s)} u_{i(t) j(t)}^{\epsilon(t)}\right) .
\end{aligned}
$$

The theorem now follows from Corollary 4.3. 
5A. Asymptotic freeness in the large dimension limit. Using Theorem 5.1, we see that the quantum groups $O_{F}^{+}$provide asymptotic models for almost-periodic free Araki-Woods factors. That is, canonical generators of almost-periodic free ArakiWoods factors can be approximated in joint distribution by normalized coordinates over a suitable sequence of $O_{F}^{+}$quantum groups.

To see this, let $\Gamma\left(H_{\mathbb{R}}, U_{t}\right)^{\prime \prime}$ be an almost-periodic free Araki-Woods factor. Then we can write $\Gamma\left(H_{\mathbb{R}}, U_{t}\right)^{\prime \prime}=\left(z_{i}: i \in \mathbb{N}\right)^{\prime \prime}$, where $\left(z_{i}\right)_{i \in \mathbb{N}}$ is a free family of $\left(1, \lambda_{i}\right)$ generalized circular elements $z_{i}$ (with $1<\lambda_{i}<\infty$ ). To approximate the joint $*$-distribution of $\left(z_{i}\right)_{i \in \mathbb{N}}$, define, for each $k \in \mathbb{N}$,

$$
F(k)=\left(\begin{array}{cc}
0 & D_{k+1}\left(1, \sqrt{\lambda_{1}}, \ldots, \sqrt{\lambda_{k}}\right)^{-1} \\
-D_{k+1}\left(1, \sqrt{\lambda_{1}}, \ldots, \sqrt{\lambda_{k}}\right) & 0
\end{array}\right) \in \mathrm{GL}_{2 k+2}(\mathbb{C}) .
$$

Theorem 5.2. The family of noncommutative random variables

$$
\left(z_{i, k}\right)_{i=1}^{k}=\left(\sqrt{N_{F(k)}} u_{1, i+1}\right)_{i=1}^{k} \subset\left(C\left(O_{F(k)}^{+}\right), h_{O_{F(k)}^{+}}\right)
$$

converges in joint distribution as $k \rightarrow \infty$ to $\left(z_{i}\right)_{i \in \mathbb{N}} \subset\left(\Gamma\left(H_{\mathbb{R}}, U_{t}\right)^{\prime \prime}, \varphi_{\Omega}\right)$.

Proof. By construction, $\left(z_{i, k}\right)_{i=1}^{k}$ and $\left(z_{i}\right)_{i=1}^{k}$ have the same left and right variances. By Theorem 5.1, we then have for any $l \in 2 \mathbb{N}, \epsilon:[l] \rightarrow\{1, *\}, i:[l] \rightarrow \mathbb{N}$, and $k=k(i)$ sufficiently large,

$$
\begin{aligned}
& \left|h_{O_{F(k)}^{+}}\left(z_{i(1), k}^{\epsilon(1)} \cdots z_{i(l), k}^{\epsilon(l)}\right)-\varphi_{\Omega}\left(z_{i(1)}^{\epsilon(1)} \cdots z_{i(l)}^{\epsilon(l)}\right)\right| \\
& \quad \leq \frac{D_{l}}{N_{F(k)}} \cdot \max _{\pi, \sigma \in \mathcal{N} \mathcal{C}_{2}(l)}\left|\delta_{\pi}^{F(k)}\left(1_{\epsilon}\right) \delta_{\sigma}^{F(k)}\left((i+1)_{\epsilon}\right)\right| \prod_{r=1}^{l}\left|t_{F(k)}(1, i(r)+1, \epsilon(r))\right| .
\end{aligned}
$$

Since both quantities

$$
\max _{\pi, \sigma \in \mathcal{N} \mathcal{C}_{2}(l)}\left|\delta_{\pi}^{F(k)}\left(1_{\epsilon}\right) \delta_{\sigma}^{F(k)}\left((i+1)_{\epsilon}\right)\right| \quad \text { and } \quad \prod_{r=1}^{l}\left|t_{F(k)}(1, i(r)+1, \epsilon(r))\right|
$$

are constant once $l, i$, and $\epsilon$ are fixed, and $N_{F(k)}=\operatorname{Tr}\left(F(k)^{*} F(k)\right) \geq \operatorname{Tr}(1)=2 k+2$, we conclude that

$$
\left|h_{O_{F(k)}^{+}}\left(z_{i(1), k}^{\epsilon(1)} \cdots z_{i(l), k}^{\epsilon(l)}\right)-\varphi_{\Omega}\left(z_{i(1)}^{\epsilon(1)} \cdots z_{i(l)}^{\epsilon(l)}\right)\right| \leq \frac{\text { constant }}{2 k+2} \rightarrow 0 .
$$

Remark 5.3. Using the same reasoning as in the proof of Theorem 5.2, it is easy to see that for the above sequence of quantum groups $\left(O_{F(k)}^{+}\right)_{k \in \mathbb{N}}$, the entire family of normalized generators

$$
\mathcal{S}_{F(k)}=\left\{\sqrt{N_{F(k)}} u_{i j}\right\}_{1 \leq i, j \leq k} \cup\left\{\sqrt{N_{F(k)}} u_{i, j+k}\right\}_{1 \leq i, j \leq k}
$$

of $C\left(O_{F(k)}^{+}\right)$converges in distribution to a free family of generalized circular elements $\left\{y_{i j}\right\}_{1 \leq i, j<\infty} \cup\left\{w_{i j}\right\}_{1 \leq i, j<\infty}$ in an NCPS $(A, \varphi)$ with the following left and 
right variances (determined by Theorem 5.1):

$$
\varphi\left(y_{i j}^{*} y_{i j}\right)=\rho_{i}^{2}, \quad \varphi\left(y_{i j} y_{i j}^{*}\right)=\rho_{j}^{-2}, \quad \varphi\left(w_{i j}^{*} w_{i j}\right)=\rho_{i}^{2}, \quad \varphi\left(w_{i j} w_{i j}^{*}\right)=\rho_{j}^{2},
$$

where $\rho_{1}=1$ and $\rho_{i}=\lambda_{i-1}^{-1 / 2}$ for $i \geq 2$. Note also that there is a state-preserving *-isomorphism $\mathrm{W}^{*}\left(\left\{y_{i j}\right\}_{1 \leq i, j<\infty} \cup\left\{w_{i j}\right\}_{1 \leq i, j<\infty}\right)$ and $\mathrm{W}^{*}\left(\left(z_{i}\right)_{i \in \mathbb{N}}\right)=\Gamma\left(H_{\mathbb{R}}, U_{t}\right)^{\prime \prime}$, the almost-periodic free Araki-Woods factor we started with. (This isomorphism follows from [Shlyakhtenko 1997, Theorem 6.4]).

5B. Asymptotic freeness in finite dimensions. In Theorem 5.2, we saw that normalized generators of a suitable family of the $O_{F}^{+}$converge in distribution to free random variables as the size $N$ of the matrices $F \in \mathrm{GL}_{N}(\mathbb{C})$ go to infinity. On the other hand, the general estimate of Theorem 5.1 shows that in the nontracial setting, the rate of approximation to freeness is governed by the growth of the quantum dimension $N_{F}=\operatorname{Tr}\left(F^{*} F\right)$, and not the physical dimension $N$. This phenomenon allows one to consider scenarios where $N_{F} \rightarrow \infty$, while the dimension $N$ of $F \in \mathrm{GL}_{N}(\mathbb{C})$ is fixed. This is illustrated by the next theorem.

Theorem 5.4. Fix $k \in \mathbb{N}$ and a sequence $\rho=\left(\rho_{1}, \ldots, \rho_{k}\right) \in(0,1)^{k}$ and let

$$
F(\gamma)=\left(\begin{array}{cccc}
0 & D_{k+1}(\rho) & 0 & 0 \\
D_{k+1}(\rho)^{-1} & 0 & 0 & 0 \\
0 & 0 & 0 & \gamma \\
0 & 0 & \gamma^{-1} & 0
\end{array}\right) \in \mathrm{GL}_{2 k+2}(\mathbb{C}) \quad(0<\gamma<1) .
$$

Then the subset of generators

$$
\tilde{\mathcal{S}}_{F(\gamma)}=\left\{\sqrt{N_{F(\gamma)}} u_{i j}\right\}_{\substack{1 \leq i \leq k \\ 1 \leq j \leq 2 k}} \subset\left(C\left(O_{F(\gamma)}^{+}\right), h_{O_{F(\gamma)}^{+}}\right)
$$

converges in distribution to a free family of generalized circular elements

$$
\tilde{\mathcal{S}}=\left\{y_{i j}\right\}_{\substack{1 \leq i \leq k \\ 1 \leq j \leq 2 k}}
$$

in a NCPS $(A, \varphi)$ with left and right variances given by

$$
\varphi\left(y_{i j}^{*} y_{i j}\right)=\rho_{i}^{2} \quad \text { and } \quad \varphi\left(y_{i j} y_{i j}^{*}\right)= \begin{cases}\rho_{j}^{-2}, & 1 \leq j \leq k, \\ \rho_{j}^{2}, & k+1 \leq j \leq 2 k .\end{cases}
$$

Remark 5.5. Note that Theorem 5.4, makes a statement about the limiting distribution of a subset $\tilde{\mathcal{S}}_{F(\gamma)}$ of generators of $C\left(O_{F(\gamma)}^{+}\right)$. We cannot make a statement about the asymptotic freeness of the entire family of generators $\mathcal{S}_{F(\gamma)}$ in this setting since some of these variables do not have limiting distributions. For example,

$$
h_{O_{F(\gamma)}^{+}}\left(\sqrt{N_{F(\gamma)}} u_{k+1, k+1}^{\epsilon(1)} \sqrt{N_{F(\gamma)}} u_{k+1, k+1}^{\epsilon(2)}\right)= \begin{cases}\gamma^{2}, & \epsilon(1)=*, \epsilon(2)=1, \\ \gamma^{-2}, & \epsilon(1)=1, \epsilon(2)=*,\end{cases}
$$

which implies that $\sqrt{N_{F(\gamma)}} u_{k+1, k+1}$ does not have a limiting distribution as $\gamma \rightarrow 0$. 
Proof of Theorem 5.4. By construction, the families $\tilde{\mathcal{S}}$ and $\tilde{\mathcal{S}}_{F(\gamma)}$ have the same left and right variances (which are independent of $\gamma$ ). Therefore, we may apply Theorem 5.1 to obtain, for any $l \in 2 \mathbb{N}, \epsilon:[l] \rightarrow\{1, *\}, i:[l] \rightarrow[k], j:[l] \rightarrow[2 k]$,

$$
\begin{aligned}
& \left|h_{O_{F(\gamma)}^{+}}\left(\sqrt{N_{F(\gamma)}} u_{i(1) j(1)}^{\epsilon(1)} \cdots \sqrt{N_{F(\gamma)}} u_{i(l) j(l)}^{\epsilon(l)}\right)-\varphi\left(y_{i(1) j(1)}^{\epsilon(1)} \cdots y_{i(l) j(l)}^{\epsilon(l)}\right)\right| \\
& \leq \frac{D_{l}}{N_{F(\gamma)}} \cdot \max _{\pi, \sigma \in \mathcal{N} \mathcal{C}_{2}(l)}\left|\delta_{\pi}^{F(\gamma)}\left(j_{\epsilon}\right) \delta_{\sigma}^{F(\gamma)}\left(i_{\epsilon}\right)\right| \prod_{r=1}^{l}\left|t_{F(\gamma)}(i(r), j(r), \epsilon(r))\right| .
\end{aligned}
$$

Since the numerator of the above expression is fixed with respect to $\gamma$ and $N_{F(\gamma)}=$ $\gamma^{2}+\gamma^{-2}+\sum_{i=1}^{k}\left(\rho_{i}^{2}+\rho_{i}^{-2}\right) \rightarrow \infty$ as $\gamma \rightarrow 0$, the theorem follows.

5C. A remark on the free unitary case. Let $c= \pm 1$ and $F \in \mathrm{GL}_{N}(\mathbb{C})$ be a canonical $F$-matrix. In this section we briefly comment on the free unitary quantum groups $U_{F}^{+}$.

In this case, it is known from the fundamental work of Banica [1997] that there is an injective $*$-homomorphism $L^{\infty}\left(U_{F}^{+}\right) \hookrightarrow L^{\infty}(\mathbb{T}) * L^{\infty}\left(O_{F}^{+}\right)$, the unital free product of $C(\mathbb{T})$ and $C\left(O_{F}^{+}\right)$, given by $v_{i j} \mapsto w u_{i j}$. Here, $w \in C(\mathbb{T})$ is canonical unitary coordinate function on the unit circle $\mathbb{T}$. Moreover, it is known that under the above embedding, $h_{U_{F}^{+}}=\left.\left(\tau * h_{O_{F}^{+}}\right)\right|_{C\left(U_{F}^{+}\right)}$, where $\tau$ denotes integration with respect to the Haar probability measure on $\mathbb{T}$.

In other words, the variables $\left\{v_{i j}\right\}_{1 \leq i, j \leq N} \subset\left(C\left(U_{F}^{+}\right), h_{U_{F}^{+}}\right)$and $\left\{w u_{i j}\right\}_{1 \leq i, j \leq N} \subset$ $\left(C(\mathbb{T}) * C\left(O_{F}^{+}\right), \tau * h_{O_{F}^{+}}\right)$are identically distributed. Using this observation, together with some basic facts about free independence and the results we have already obtained on $O_{F}^{+}$, we arrive at the following unitary version of Theorem 5.1, whose proof we leave as an exercise to the reader. (Note that the extra freeness inside $C\left(U_{F}^{+}\right)$given by the above free product model yields a slightly cleaner statement than Theorem 5.1.)

Theorem 5.6. Fix a canonical deformation matrix

$$
F=F_{\rho}^{(c)}=\left(\begin{array}{ccc}
0 & D_{k}(\rho) & 0 \\
c D_{k}(\rho)^{-1} & 0 & 0 \\
0 & 0 & 1_{N-2 k}
\end{array}\right) \in \mathrm{GL}_{N}(\mathbb{C})
$$

and let $\mathcal{S}=\left\{y_{i j}\right\}_{1 \leq i, j \leq N}$ be a family of noncommutative random variables in an $\operatorname{NCPS}(A, \varphi)$ with the following properties.

(1) $\mathcal{S}$ is freely independent.

(2) For each $i, j$, the elements $y_{i j} \in \mathcal{S}$ and $\sqrt{N_{F}} v_{i j} \in C\left(U_{F}^{+}\right)$have the same left and right variances, given by the matrix entries of $N_{F} \Phi$ in (3-9).

(3) Each $y_{i j}$ is a generalized circular element. 
Then for each $l \in 2 \mathbb{N}$, there is a constant $D_{l}>0$ such that

$$
\begin{aligned}
\mid h_{U_{F}^{+}}\left(\sqrt{N_{F}} v_{i(1) j(1)}^{\epsilon(1)} \cdots\right. & \left.\sqrt{N_{F}} v_{i(l) j(l)}^{\epsilon(l)}\right)-\varphi\left(y_{i(1) j(1)}^{\epsilon(1)} \cdots y_{i(l) j(l)}^{\epsilon(l)}\right) \mid \\
\leq & \frac{D_{l}}{N_{F}} \max _{\pi, \sigma \in \mathcal{N} \mathcal{C}_{2}(l)}\left|\delta_{\pi}^{F}\left(j_{\epsilon}\right) \delta_{\sigma}^{F}\left(i_{\epsilon}\right)\right| \prod_{r=1}^{l}\left|t_{F}(i(r), j(r), \epsilon(r))\right|,
\end{aligned}
$$

for each $\epsilon:[l] \rightarrow\{1, *\}$ and $i, j:[l] \rightarrow[N]$.

Remark 5.7. The most general version of Theorem 5.6 for $U_{F}^{+}$with $F \in \mathrm{GL}_{N}(\mathbb{C})$ arbitrary is not known to the authors and requires further investigation.

\section{6. $O_{F}^{+}$and quantum symmetries of free Araki-Woods factors}

In this final section we return to the free orthogonal quantum groups $O_{F}^{+}$and investigate to what extent they can be regarded as quantum symmetries of free ArakiWoods factors. Inspired by the fact that the free group factors $L\left(\mathbb{F}_{N}\right)$ admit $O_{N}^{+}$as natural quantum symmetries [Curran 2010], we are interested in finding canonical families of (nontracial) noncommutative random variables $\left(x_{1}, \ldots, x_{N}\right)$ belonging to an NCPS $(A, \varphi)$ whose joint distribution is $O_{F}^{+}$-invariant in the following sense.

Definition 6.1. Let $(A, \varphi)$ be an NCPS and consider $\boldsymbol{x}=\left(x_{1}, \ldots, x_{N}\right) \subset A$. Fix $F \in$ $\mathrm{GL}_{N}(\mathbb{C})$ and let $U=\left[u_{i j}\right] \in M_{N}\left(C\left(O_{F}^{+}\right)\right)$be the fundamental representation of $O_{F}^{+}$. We say that $\boldsymbol{x}$ has an $O_{F}^{+}$-invariant joint distribution (or, that $\boldsymbol{x}$ is $O_{F}^{+}$-rotatable) if for any $l \in \mathbb{N}, i:[l] \rightarrow[N]$, and any $\epsilon:[l] \rightarrow\{1, *\}$,

$$
\begin{aligned}
\sum_{j:[l] \rightarrow N} u_{i(1) j(1)}^{\epsilon(1)} u_{i(2) j(2)}^{\epsilon(2)} \cdots u_{i(l) j(l)}^{\epsilon(l)} \varphi\left(x_{j(1)}^{\epsilon(1)} x_{j(2)}^{\epsilon(2)} \cdots\right. & \left.x_{j(l)}^{\epsilon(l)}\right) \\
& =\varphi\left(x_{i(1)}^{\epsilon(1)} x_{i(2)}^{\epsilon(2)} \cdots x_{i(l)}^{\epsilon(l)}\right) 1 .
\end{aligned}
$$

We note that the existence of an $N$-tuple $x$ with the above $O_{F}^{+}$-invariance property is connected to the existence of an action of the quantum group $O_{F}^{+}$on the von Neumann algebra generated by $\boldsymbol{x}$.

Definition 6.2. Let $\mathbb{G}$ be a compact quantum group with von Neumann algebra $L^{\infty}(\mathbb{G})=\pi_{h}(C(\mathbb{G}))^{\prime \prime}$ and (extended) coproduct $\Delta_{r}: L^{\infty}(\mathbb{G}) \rightarrow L^{\infty}(\mathbb{G}) \bar{\otimes} L^{\infty}(\mathbb{G})$. Let $M$ a von Neumann algebra.

(1) A left action (or simply an action) of $\mathbb{G}$ on $M$ is a normal, injective, and unital *-homomorphism $\alpha: M \rightarrow L^{\infty}(\mathbb{G}) \bar{\otimes} M$ such that $(\iota \otimes \alpha) \circ \alpha=(\Delta \otimes \iota) \circ \alpha$. We denote the action of $\mathbb{G}$ on $M$ by the notation $\mathbb{G} \curvearrowright^{\alpha} M$.

(2) If $\varphi$ is a faithful normal state on $M$, we say that an action $\mathbb{G} \curvearrowright^{\alpha} M$ is $\varphi$-preserving if $(\iota \otimes \varphi) \circ \alpha=\varphi(\cdot) 1_{L^{\infty}(\mathbb{G})}$. Such an action has notation $\mathbb{G} \curvearrowright^{\alpha}(M, \varphi)$. 
Remark 6.3. If $\boldsymbol{x}=\left(x_{1}, \ldots, x_{N}\right) \subset(A, \varphi)$ is an $N$-tuple with an $O_{F}^{+}$-invariant joint distribution, then it is easy to see that

$$
\alpha\left(x_{i}\right)=\sum_{j=1}^{N} \pi_{h}\left(u_{i j}\right) \otimes x_{j} \quad(1 \leq i \leq n)
$$

defines a $\varphi$-preserving action $O_{F}^{+} \curvearrowright^{\alpha}\left(\mathrm{W}^{*}\left(x_{1}, \ldots, x_{N}\right), \varphi\right)$, where $\pi_{h}: C\left(O_{F}^{+}\right) \rightarrow$ $L^{\infty}\left(O_{F}^{+}\right)$is the GNS representation associated to the Haar state.

We now show that such $O_{F}^{+}$-invariant noncommutative random variables exist for any $F$. To do this, we first need a lemma.

Lemma 6.4. Let $Q=F^{t} \bar{F}$, where $F \in \mathrm{GL}_{N}(\mathbb{C})$ is a canonical $F$ matrix (so that, in particular, $Q$ is diagonal $)$, and let $U=\left[u_{i j}\right] \in M_{N}\left(C\left(O_{F}^{+}\right)\right)$be the fundamental representation of $O_{F}^{+}$. Then

$$
\sum_{r=1}^{N} u_{i r} u_{j r}^{*}=\delta_{i j} 1 \quad \text { and } \quad \sum_{r=1}^{N} u_{i r}^{*} u_{j r}\left(Q^{-1}\right)_{r r}=\delta_{i j}\left(Q^{-1}\right)_{i i} 1 \quad(1 \leq i, j \leq N) .
$$

Proof. The first equality is a direct consequence of the fact that the fundamental representation $U$ is unitary. To prove the second inequality, we use [Timmermann 2008, Proposition 3.2.17] which shows that $\bar{U} \bar{Q}^{-1} U^{t}=\bar{Q}^{-1}$. Since $Q$ is diagonal and positive definite, the result follows.

In the case where $F \in \mathrm{GL}_{N}(\mathbb{C})$ is canonical, the way to find $O_{F}^{+}$-rotatable generators of a free Araki-Woods factor is to fix a column of the fundamental representation of $O_{F}^{+}$and take an $N$-tuple of freely independent generalized circular elements with the same left and right variances as this column (up to a common nonzero scaling factor). Again, we note that after completion of a first draft of this paper, it was pointed out to the authors that the following theorem can also be obtained as a consequence of [Vaes 2005, Proposition 3.1].

Theorem 6.5. Let $F \in \mathrm{GL}_{N}(\mathbb{C})$ be a canonical $F$ matrix and let $Q=F^{t} \bar{F}$ with diagonal entries $\left(Q_{i i}\right)_{i=1}^{N}$. Let $\boldsymbol{x}=\left(x_{1}, \ldots, x_{N}\right) \subset(A, \varphi)$ be a $*$-free family of generalized circular elements with left and right covariances given by

$$
\varphi\left(x_{i}^{*} x_{i}\right)=Q_{i i}^{-1}, \quad \varphi\left(x_{i} x_{i}^{*}\right)=1, \quad(1 \leq i \leq N) .
$$

Then $\boldsymbol{x}$ has an $O_{F}^{+}$-invariant joint $*$-distribution. In other words, there is a $\varphi$-preserving action $O_{F}^{+} \curvearrowright^{\alpha}\left(\mathrm{W}^{*}\left(x_{1}, \ldots, x_{N}\right), \varphi\right)$ given by

$$
\alpha\left(x_{i}\right)=\sum_{j=1}^{N} \pi_{h}\left(u_{i j}\right) \otimes x_{j}
$$

where $U=\left[u_{i j}\right]$ is the fundamental representation of $O_{F}^{+}$. 
Proof. We must verify (6-1) for the $N$-tuple $\boldsymbol{x}$, for each choice of $l \in \mathbb{N}, i:[l] \rightarrow[N]$, and $\epsilon:[l] \rightarrow\{1, *\}$. To start, observe that when $l$ is odd or $\left|\epsilon^{-1}(1)\right| \neq\left|\epsilon^{-1}(*)\right|$, then both sides of (6-1) are always zero. Therefore, we assume $l \in 2 \mathbb{N}$ and that $\left|\epsilon^{-1}(1)\right|=\left|\epsilon^{-1}(*)\right|=l / 2$.

We begin by considering the case $l=2$, and fix $1 \leq i(1), i(2) \leq N, \epsilon(1) \neq \epsilon(2) \in$ $\{1, *\}$. Then we have

$$
\sum_{j(1), j(2)=1}^{N} u_{i(1) j(1)}^{\epsilon(1)} u_{i(2) j(2)}^{\epsilon(2)} \varphi\left(x_{j(1)}^{\epsilon(1)} x_{j(2)}^{\epsilon(2)}\right)=\sum_{j(1)=1}^{N} u_{i(1) j(1)}^{\epsilon(1)} u_{i(2) j(1)}^{\epsilon(2)} \varphi\left(x_{j(1)}^{\epsilon(1)} x_{j(1)}^{\epsilon(2)}\right) .
$$

If $\epsilon(1)=1$ and $\epsilon(2)=*$, then $\varphi\left(x_{j(1)} x_{j(1)}^{*}\right)=1$ and the above quantity equals

$$
\sum_{j(1)=1}^{N} u_{i(1) j(1)} u_{i(2) j(1)}^{*}=\delta_{i(1), i(2)} 1=\varphi\left(x_{i(1)} x_{i(2)}^{*}\right) 1
$$

by Lemma 6.4. If $\epsilon(1)=*$ and $\epsilon(2)=1$, then $\varphi\left(x_{j(1)}^{*} x_{j(1)}\right)=Q_{j(1) j(1)}^{-1}$ and the above quantity equals

$$
\sum_{j(1)=1}^{N} u_{i(1) j(1)}^{*} u_{i(2) j(1)} Q_{j(1) j(1)}^{-1}=\delta_{i(1), i(2)} \varphi\left(x_{i(1)}^{*} x_{i(1)}\right) 1=\varphi\left(x_{i(1)}^{*} x_{i(2)}\right) 1,
$$

again by Lemma 6.4. In each case, we obtain

$$
\sum_{j(1), j(2)=1}^{N} u_{i(1) j(1)}^{\epsilon(1)} u_{i(2) j(2)}^{\epsilon(2)} \varphi\left(x_{j(1)}^{\epsilon(1)} x_{j(2)}^{\epsilon(2)}\right)=\varphi\left(x_{i(1)}^{\epsilon(1)} x_{i(2)}^{\epsilon(2)}\right) 1 .
$$

Now let $2<l \in 2 \mathbb{N}$ and fix $\epsilon:[l] \rightarrow\{1, *\}$ and $i:[l] \rightarrow[N]$. Then from Theorem 2.2,

$$
\begin{aligned}
\sum_{j:[l] \rightarrow N} u_{i(1) j(1)}^{\epsilon(1)} u_{i(2) j(2)}^{\epsilon(2)} \cdots u_{i(l) j(l)}^{\epsilon(l)} \varphi\left(x_{j(1)}^{\epsilon(1)} x_{j(2)}^{\epsilon(2)} \cdots x_{j(l)}^{\epsilon(l)}\right) & \\
& =\sum_{j:[l] \rightarrow N} u_{i(1) j(1)}^{\epsilon(1)} u_{i(2) j(2)}^{\epsilon(2)} \cdots u_{i(l) j(l)}^{\epsilon(l)}\left(\sum_{\pi \in \mathcal{N} \mathcal{C}_{2}^{\epsilon}(l)} \prod_{(s, t) \in \pi} \varphi\left(x_{j(s)}^{\epsilon(s)} x_{j(t)}^{\epsilon(t)}\right)\right) \\
& =\sum_{\pi \in \mathcal{N} \mathcal{C}_{2}^{\epsilon(l)}}\left(\sum_{j:[l] \rightarrow N} u_{i(1) j(1)}^{\epsilon(1)} u_{i(2) j(2)}^{\epsilon(2)} \cdots u_{i(l) j(l)}^{\epsilon(l)} \prod_{(s, t) \in \pi} \varphi\left(x_{j(s)}^{\epsilon(s)} x_{j(t)}^{\epsilon(t)}\right)\right) .
\end{aligned}
$$

Fix $\pi \in \mathcal{N C}_{2}^{\epsilon}(l)$ and consider the internal sum above. Since $\pi$ is noncrossing, it contains a neighboring pair $(r, r+1)$. Applying (6-2) to the partial sum over 
$1 \leq j(r), j(r+1) \leq N$, we obtain

$$
\begin{aligned}
& \sum_{j:[l] \rightarrow[N]} u_{i(1) i(1)}^{\epsilon(1)} u_{i(2) i(2)}^{\epsilon(2)} \cdots u_{i(l) i(l)}^{\epsilon(l)} \prod_{(s, t) \in \pi} \varphi\left(x_{j(s)}^{\epsilon(s)} x_{j(t)}^{\epsilon(t)}\right) \\
& =\varphi\left(x_{i(r)}^{\epsilon(r)} x_{i(r+1)}^{\epsilon(r+1)}\right) \\
& \quad \times\left(\sum_{j:[l] \backslash\{r, r+1\} \rightarrow[N]} u_{i(1) i(1)}^{\epsilon(1)} \cdots u_{i(r-1) i(r-1)}^{\epsilon(r-1)} u_{i(r+2) i(r+2)}^{\epsilon(r+2)} \cdots u_{i(l) i(l)}^{\epsilon(l)} \prod_{(s, t) \in \mathcal{\pi} \backslash(r, r+1)} \varphi\left(x_{j(s)}^{\epsilon(s)} x_{j(t)}^{\epsilon(t)}\right)\right) .
\end{aligned}
$$

Repeatedly applying the same principle to this new internal sum of lower order (note that $\pi \backslash(r, r+1)$ is again noncrossing), after a total of $l / 2-1$ steps, we get

$$
\sum_{j:[l] \rightarrow[N]} u_{i(1) i(1)}^{\epsilon(1)} u_{i(2) i(2)}^{\epsilon(2)} \cdots u_{i(l) i(l)}^{\epsilon(l)} \prod_{(s, t) \in \pi} \varphi\left(x_{j(s)}^{\epsilon(s)} x_{j(t)}^{\epsilon(t)}\right)=\prod_{(s, t) \in \pi} \varphi\left(x_{i(s)}^{\epsilon(s)} x_{i(t)}^{\epsilon(t)}\right) 1 .
$$

Therefore,

$$
\begin{aligned}
\sum_{j:[l] \rightarrow N} u_{i(1) j(1)}^{\epsilon(1)} u_{i(2) j(2)}^{\epsilon(2)} & \cdots u_{i(l) j(l)}^{\epsilon(l)} \varphi\left(x_{j(1)}^{\epsilon(1)} x_{j(2)}^{\epsilon(2)} \cdots x_{j(l)}^{\epsilon(l)}\right) \\
& =\sum_{\pi \in \mathcal{N} C_{2}^{\epsilon}(l)} \prod_{(s, t) \in \pi} \varphi\left(x_{i(s)}^{\epsilon(s)} x_{i(t)}^{\epsilon(t)}\right) 1=\varphi\left(x_{i(1)}^{\epsilon(1)} x_{i(2)}^{\epsilon(2)} \cdots x_{i(l)}^{\epsilon(l)}\right) 1 .
\end{aligned}
$$

Remark 6.6. It is clear that $\left(\mathrm{W}^{*}\left(x_{1}, \ldots, x_{N}\right), \varphi\right) \cong\left(\Gamma\left(\mathcal{H}_{\mathbb{R}}, U_{t}\right)^{\prime \prime}, \varphi_{\Omega}\right)$ is a free Araki-Woods factor associated to a finite dimensional orthogonal representation $\left(U_{t}\right)_{r \in \mathbb{R}}$ (compare with Theorem 2.3). Moreover, it is interesting to note that the type classification for the von Neumann algebras $L^{\infty}\left(O_{F}^{+}\right)$and $\Gamma\left(H_{\mathbb{R}}, U_{t}\right)^{\prime \prime}$ is the same. More precisely, if $\Gamma<\mathbb{R}_{+}^{*}$ is the subgroup generated by the eigenvalues of $Q \otimes Q^{-1}$, then both of these algebras are type $\mathrm{II}_{1}$ when $Q=1$, type $\mathrm{III}_{\lambda}$ if $\Gamma=\lambda^{\mathbb{Z}}$, and type III $_{1}$ otherwise. Compare [Shlyakhtenko 1997, Theorem 6.1; Vaes and Vergnioux 2007, Theorem 7.1].

Remark 6.7. Theorem 6.5 only considers the case of a canonical matrix $F$. For generic $F \in \mathrm{GL}_{N}(\mathbb{C})$ such that $F \bar{F}=c 1$, recall from Section 3A that there is a canonical $F$-matrix $F_{\rho}^{(c)} \in \mathrm{GL}_{N}(\mathbb{C})$ and $v \in \mathcal{U}_{N}$ such that $F_{\rho}^{(c)}=v F v^{t}$ and $O_{F}^{+} \cong O_{F_{\rho}^{(c)}}^{+}$. Then $O_{F}^{+} \curvearrowright^{\alpha_{F}}\left(\Gamma\left(\mathcal{H}_{\mathbb{R}}, U_{t}\right)^{\prime \prime}, \varphi_{\Omega}\right)$, where $\Gamma\left(\mathcal{H}_{\mathbb{R}}, U_{t}\right)^{\prime \prime}$ is the free ArakiWoods factor on which $O_{F_{\rho}^{(c)}}^{+}$acts in the sense of the above theorem. Indeed let $\boldsymbol{x}=\left(x_{1}, \ldots, x_{N}\right)$ be the generalized circular system constructed in Theorem 6.5, let $\alpha_{F_{\rho}^{(c)}}$ be the corresponding action, and let $\boldsymbol{y}=v \boldsymbol{x}$. Then $\mathrm{W}^{*}(\boldsymbol{x})=\mathrm{W}^{*}(\boldsymbol{y})$ and one readily checks from the defining relations that

$$
W=v U v^{*}
$$

where $W=\left[w_{i j}\right]$ and $U=\left[u_{i j}\right]$ are the fundamental representations of $O_{F}^{+}$and $O_{F_{\rho}^{(c)}}^{+}$, respectively. A simple calculation then shows that condition (6-1) holds with the $w_{i j}$ replacing the $u_{i j}$ and the $y_{i}$ replacing the $x_{i}$. 


\section{Acknowledgments}

Brannan was partially supported by an NSERC Postdoctoral Fellowship. Kirkpatrick was partially supported by NSF Grant DMS-1106770 and NSF CAREER Award DMS-1254791. Both Brannan and Kirkpatrick wish to thank Todd Kemp for encouraging them to collaborate.

\section{References}

[Banica 1996] T. Banica, "Théorie des représentations du groupe quantique compact libre $\mathrm{O}(n)$ ", $C$. R. Acad. Sci. Paris Sér. I Math. 322:3 (1996), 241-244. MR 97a:46108 Zbl 0862.17010

[Banica 1997] T. Banica, "Le groupe quantique compact libre U(n)", Comm. Math. Phys. 190:1 (1997), 143-172. MR 99k:46095 Zbl 0906.17009

[Banica and Collins 2007] T. Banica and B. Collins, "Integration over compact quantum groups", Publ. Res. Inst. Math. Sci. 43:2 (2007), 277-302. MR 2008m:46137 Zbl 1129.46058

[Banica et al. 2009] T. Banica, B. Collins, and P. Zinn-Justin, "Spectral analysis of the free orthogonal matrix", Int. Math. Res. Not. 2009:17 (2009), 3286-3309. MR 2011d:46133 Zbl 1179.46056

[Bichon et al. 2006] J. Bichon, A. De Rijdt, and S. Vaes, "Ergodic coactions with large multiplicity and monoidal equivalence of quantum groups", Comm. Math. Phys. 262:3 (2006), 703-728. MR 2007a:46072 Zbl 1122.46046

[Collins 2003] B. Collins, "Moments and cumulants of polynomial random variables on unitary groups, the Itzykson-Zuber integral, and free probability", Int. Math. Res. Not. 2003:17 (2003), 953-982. MR 2003m:28015 Zbl 1049.60091

[Collins and Śniady 2006] B. Collins and P. Śniady, "Integration with respect to the Haar measure on unitary, orthogonal and symplectic group", Comm. Math. Phys. 264:3 (2006), 773-795. MR 2007c:60009 Zbl 1108.60004

[Curran 2010] S. Curran, "Quantum rotatability", Trans. Amer. Math. Soc. 362:9 (2010), 4831-4851. MR 2011e:46105 Zbl 1203.46043

[De Commer et al. 2014] K. De Commer, A. Freslon, and M. Yamashita, "CCAP for universal discrete quantum groups", Comm. Math. Phys. 331:2 (2014), 677-701. MR 3238527 Zbl 06346288

[Diaconis and Freedman 1987] P. Diaconis and D. Freedman, "A dozen de Finetti-style results in search of a theory", Ann. Inst. H. Poincaré Probab. Statist. 23:2, suppl. (1987), 397-423. MR 88f:60072 Zbl 0619.60039

[Freedman 1962] D. A. Freedman, "Invariants under mixing which generalize de Finetti's theorem", Ann. Math. Statist 33 (1962), 916-923. MR 27 \#6292 Zbl 0201.49501

[Kallenberg 2005] O. Kallenberg, Probabilistic symmetries and invariance principles, Springer, New York, 2005. MR 2006i:60002 Zbl 1084.60003

[Nica and Speicher 2006] A. Nica and R. Speicher, Lectures on the combinatorics of free probability, London Mathematical Society Lecture Note Series 335, Cambridge Univ. Press, 2006. MR 2008k:46198 Zbl 1133.60003

[Shlyakhtenko 1997] D. Shlyakhtenko, "Free quasi-free states", Pacific J. Math. 177:2 (1997), 329368. MR 98b:46086 Zbl 0882.46026

[Timmermann 2008] T. Timmermann, An invitation to quantum groups and duality: From Hopf algebras to multiplicative unitaries and beyond, European Mathematical Society (EMS), Zürich, 2008. MR 2009f:46079 Zbl 1162.46001 
[Vaes 2005] S. Vaes, "Strictly outer actions of groups and quantum groups", J. Reine Angew. Math. 578 (2005), 147-184. MR 2005k:46167 Zbl 1073.46047

[Vaes and Vergnioux 2007] S. Vaes and R. Vergnioux, "The boundary of universal discrete quantum groups, exactness, and factoriality”, Duke Math. J. 140:1 (2007), 35-84. MR 2010a:46166 Zbl 1129.46062

[Van Daele and Wang 1996] A. Van Daele and S. Wang, "Universal quantum groups", Internat. J. Math. 7:2 (1996), 255-263. MR 97d:46090 Zbl 0870.17011

[Wang 1993] S. Wang, General constructions of compact quantum groups, Ph.D. thesis, University of California, Berkeley, 1993, Available at http://search.proquest.com/docview/304078126.

[Wang 2002] S. Wang, "Structure and isomorphism classification of compact quantum groups $A_{u}(Q)$ and $B_{u}(Q)$ ", J. Operator Theory 48:3, suppl. (2002), 573-583. MR 2004b:46083 Zbl 1029.46089

[Weingarten 1978] D. Weingarten, "Asymptotic behavior of group integrals in the limit of infinite rank”, J. Mathematical Phys. 19:5 (1978), 999-1001. MR 57 \#11421 Zbl 0388.28013

[Woronowicz 1987] S. L. Woronowicz, "Compact matrix pseudogroups", Comm. Math. Phys. 111:4 (1987), 613-665. MR 88m:46079 Zbl 0627.58034

[Woronowicz 1998] S. L. Woronowicz, “Compact quantum groups”, pp. 845-884 in Symétries quantiques (Les Houches, 1995), edited by A. Connes et al., North-Holland, Amsterdam, 1998. MR 99m:46164 Zbl 0997.46045

Received June 19, 2015.

MiCHAEL BRANNAN

DEPARTMENT OF MATHEMATICS

TEXAS A\&M UNIVERSITY

College Station, TX 77843

UNITED STATES

mbrannan@math.tamu.edu

KAY KIRKPATRICK

UNIVERSITY OF ILLINOIS AT URBANA-CHAMPAIGN

URBANA, IL 61821

UNITED STATES

kkirkpat@illinois.edu 


\title{
PACIFIC JOURNAL OF MATHEMATICS
}

\author{
msp.org/pjm
}

Founded in 1951 by E. F. Beckenbach (1906-1982) and F. Wolf (1904-1989)

\section{EDITORS}

Don Blasius (Managing Editor)

Department of Mathematics

University of California

Los Angeles, CA 90095-1555

blasius@math.ucla.edu

\author{
Paul Balmer \\ Department of Mathematics \\ University of California \\ Los Angeles, CA 90095-1555 \\ balmer@math.ucla.edu \\ Robert Finn \\ Department of Mathematics \\ Stanford University \\ Stanford, CA 94305-2125 \\ finn@math.stanford.edu \\ Sorin Popa \\ Department of Mathematics \\ University of California \\ Los Angeles, CA 90095-1555 \\ popa@math.ucla.edu
}

\author{
Vyjayanthi Chari \\ Department of Mathematics \\ University of California \\ Riverside, CA 92521-0135 \\ chari@math.ucr.edu \\ Kefeng Liu \\ Department of Mathematics \\ University of California \\ Los Angeles, CA 90095-1555 \\ liu@math.ucla.edu \\ Jie Qing \\ Department of Mathematics \\ University of California \\ Santa Cruz, CA 95064 \\ qing@ cats.ucsc.edu
}

\section{PRODUCTION}

Silvio Levy, Scientific Editor, production@msp.org

\section{SUPPORTING INSTITUTIONS}

ACADEMIA SINICA, TAIPEI

CALIFORNIA INST. OF TECHNOLOGY

INST. DE MATEMÁTICA PURA E APLICADA

KEIO UNIVERSITY

MATH. SCIENCES RESEARCH INSTITUTE

NEW MEXICO STATE UNIV.

OREGON STATE UNIV.

\author{
STANFORD UNIVERSITY \\ UNIV. OF BRITISH COLUMBIA \\ UNIV. OF CALIFORNIA, BERKELEY \\ UNIV. OF CALIFORNIA, DAVIS \\ UNIV. OF CALIFORNIA, LOS ANGELES \\ UNIV. OF CALIFORNIA, RIVERSIDE \\ UNIV. OF CALIFORNIA, SAN DIEGO \\ UNIV. OF CALIF., SANTA BARBARA
}

\author{
Daryl Cooper \\ Department of Mathematics \\ University of California \\ Santa Barbara, CA 93106-3080 \\ cooper@math.ucsb.edu \\ Jiang-Hua Lu \\ Department of Mathematics \\ The University of Hong Kong \\ Pokfulam Rd., Hong Kong \\ jhlu@maths.hku.hk \\ Paul Yang \\ Department of Mathematics \\ Princeton University \\ Princeton NJ 08544-1000 \\ yang@math.princeton.edu
}

These supporting institutions contribute to the cost of publication of this Journal, but they are not owners or publishers and have no responsibility for its contents or policies.

See inside back cover or msp.org/pjm for submission instructions.

The subscription price for 2016 is US $\$ 440 /$ year for the electronic version, and $\$ 600 /$ year for print and electronic.

Subscriptions, requests for back issues and changes of subscribers address should be sent to Pacific Journal of Mathematics, P.O. Box 4163, Berkeley, CA 94704-0163, U.S.A. The Pacific Journal of Mathematics is indexed by Mathematical Reviews, Zentralblatt MATH, PASCAL CNRS Index, Referativnyi Zhurnal, Current Mathematical Publications and Web of Knowledge (Science Citation Index).

The Pacific Journal of Mathematics (ISSN 0030-8730) at the University of California, c/o Department of Mathematics, 798 Evans Hall \#3840, Berkeley, CA 94720-3840, is published twelve times a year. Periodical rate postage paid at Berkeley, CA 94704, and additional mailing offices. POSTMASTER: send address changes to Pacific Journal of Mathematics, P.O. Box 4163, Berkeley, CA 94704-0163.

PJM peer review and production are managed by EditFLOW ${ }^{\circledR}$ from Mathematical Sciences Publishers.

\section{PUBLISHED BY}

\section{mathematical sciences publishers \\ nonprofit scientific publishing}

http://msp.org/

(C) 2016 Mathematical Sciences Publishers 


\section{PACIFIC JOURNAL OF MATHEMATICS}

Volume 282 No. $1 \quad$ May 2016

On the half-space theorem for minimal surfaces in Heisenberg space

Tristan Alex

Extending smooth cyclic group actions on the Poincaré homology sphere 9

NIMA ANVARI

A short proof of the existence of supercuspidal representations for all reductive $p$-adic groups

RAPHAËL BEUZART-PLESSIS

Quantum groups and generalized circular elements

MICHAEL BRANNAN and KAY KIRKPATRICK

Volumes of Montesinos links

KathleEn Finlinson and Jessica S. PurCell

Minimal surfaces with two ends which have the least total absolute curvature

SHOICHI FUJIMORI and TOSHIHIRO SHODA

Multiplicité du spectre de Steklov sur les surfaces et nombre chromatique

\section{PIERRE JAMMES}

$E$-polynomial of the $\mathrm{SL}(3, \mathbb{C})$-character variety of free groups

SEAN LAWTON and VicEnTE MuÑOZ

The Blum-Hanson property for $\mathscr{C}(K)$ spaces

PASCAL LEFÈVRE and ÉTIENNE MATHERON

Crossed product algebras and direct integral decomposition for Lie supergroups

KARL-HERMANN NEEB and HADI SALMASIAN Associated primes of local cohomology modules over regular rings

TONY J. PUTHENPURAKAL 